\title{
ON CONDITIONING OF A SCHWARZ METHOD FOR SINGULARLY PERTURBED CONVECTION-DIFFUSION EQUATIONS IN THE CASE OF DISTURBANCES IN THE DATA OF THE BOUNDARY VALUE PROBLEM ${ }^{1}$
}

\author{
GRIGORII I. SHISHKIN \\ Institute of Mathematics and Mechanics, Ural Branch of Russian Academy of Science \\ 16 S. Kovalevskaya St., 620219 Ekaterinburg, Russia \\ E-mail: shishkin@imm.uran.ru
}

\begin{abstract}
In this paper we consider a Dirichlet problem for singularly perturbed ordinary differential equations with convection terms and a small perturbation parameter $\varepsilon$. To solve the problem numerically we use an $\varepsilon$-uniformly convergent difference scheme (on special piecewise-uniform meshes) and a decomposition of this scheme based on a Schwarz technique with overlapping subdomains. The step-size of such special meshes is extremely small in a neighbourhood of the layer and changes sharply on its boundary, which can generally lead to a loss of conditioning of the above schemes. We study the influence of perturbations in the data of the boundary value problem on disturbances of numerical solutions. We derive estimates for the disturbances of numerical solutions (in the maximum norm) depending on a subdomain in which the disturbance of the data appears. When the right-hand side of the discrete equations is considered in a "natural" norm, i.e., in the maximum norm with a special weight multiplier (that is $\varepsilon \ln N$, for $\varepsilon=\mathcal{O}\left(\ln ^{-1} N\right)$, in a neighbourhood of the boundary layer, where $N$ defines the number of mesh points), the finite difference schemes under consideration are well conditioned $\varepsilon$-uniformly. In addition, for the Schwarz method a special restriction is imposed on the width of the overlapping region. Note that for these special schemes an $\varepsilon$-uniform estimate for the condition number is the same as that for schemes on uniform meshes in the case of regular boundary value problems. We give conditions under which the solution of the iterative scheme based on the overlapping Schwarz method is convergent $\varepsilon$-uniformly to the solution of the Dirichlet problem as the number of mesh points and the number of iterations increase.
\end{abstract}

2000 Mathematics Subject Classification: 65M06; 65M12; 65M15.

Key words: singular perturbation problem, convection-diffusion equations, $\varepsilon$-uniform fitted mesh method, domain decomposition method, condition numbers.

\section{Introduction}

The difficulties are well known which arise when we find a numerical solution of singularly perturbed boundary value problems, i.e., boundary value problems for equations whose higher-

\footnotetext{
${ }^{1}$ This research was supported in part by the Dutch Research Organisation NWO under grant N 047.008.007, Enterprise Ireland Research Grant SC-2000-070 and by the Russian Foundation for Basic Research under grant N 01-01-01022.
} 
order derivatives contain a small perturbation parameter $\varepsilon$ (see, e.g., [1]-[6] and also the bibliography therein). The errors of numerical solutions obtained with the use of traditional schemes turn out to be too large (compared with the exact solutions) for small values of the parameter (see, e.g., estimate (3.14a) in the case of problem (2.1), (2.2) for an ordinary differential convection-diffusion equation, Section 3). Therefore it is necessary to contsruct special numerical methods whose accuracy does not depend on the value of the parameter $\varepsilon$ (i.e., $\varepsilon$-uniformly convergent methods). A number of such special methods use meshes which are condensed in a boundary layer (see the description of these methods, e.g., in [1], [4]-[6]), the step-size of such meshes in a neighbourhood of the boundary layer is much less than the parameter $\varepsilon$. In $[4,6]$ (see also the references in [4]-[6]) piecewise uniform meshes with an abrupt change in the mesh-size are used; the mesh-size of such meshes is extremely small in the neighbourhood of the boundary layers. These properties can lead to high sensitivity of the numerical solution to computation errors, in particular, to a loss in conditioning of the difference scheme for small values of the parameter. As was shown by A.A. Samarskii [7], even for regular boundary value problems well conditioning of a scheme fails on a nonuniform mesh when the ratio of the fine and coarse mesh sizes tends to zero.

In the case of domain decomposition methods used for solving singularly perturbed problems, the rate of convergence of the iterative process depends both on the parameter $\varepsilon$ and on a big variety of parameters of the method and can be very small for the method to be of practical use (for example, the iterative process in the case of minimal overlapping subdomains converges, as the number $n$ of iterations grows, at a rate $O\left(q^{n}\right)$ of the geometrical progression with degree $q \leq 1-m N^{-1}$, where $N$ defines the number of mesh points; see estimate (3.20) in Section 3). It can turn out that the solution of a decomposition scheme does not converge to the solution of the base scheme subject to decomposition as the number of mesh points and the number of iterations grow (see, for example, the results of Section 3, Subsection 3 in the case of the decomposition scheme (2.6), (3.8) for $N, n \rightarrow \infty)$.

The errors in the solutions of iterative methods due to perturbation of the data can be accumulated and distort essentially the numerical solution (in the case of systems of linear equations see, e.g.,[10]).

Because of these reasons, in the case of singularly perturbed boundary value problems it is of great interest to find conditions for the $\varepsilon$-uniform convergence of iterative methods (for $N$, $n \rightarrow \infty$ ), and also to study the influence of the disturbances of the data on the behaviour of the numerical solution. It is of interest to investigate the questions of conditioning of iterative methods.

In the present paper we consider the first boundary value problem for singularly perturbed ordinary differential equations with convective terms. To approximate the problem, we use a classical monotone difference scheme on special piecewise uniform meshes condensing in a boundary layer [4]; this (base) difference scheme converges $\varepsilon$-uniformly. We study how the perturbations in the data of the boundary value problem influence the behaviour of numerical solutions of the base scheme and of the decomposition scheme, in particular, for arbitrarily small values of $\varepsilon$.

It is shown that the condition number is $\varepsilon$-uniformly bounded (see Theorem 3.1, Section 3) on uniform meshes or on meshes close to uniform ones (schemes on such meshes do not converge $\varepsilon$-uniformly; see estimate (3.14a)). However, for $\varepsilon$-uniformly convergent schemes on piecewise uniform meshes the condition number of difference operators (in the classical sense) is not $\varepsilon$-uniformly bounded (see Theorem 3.2). This behaviour of the condition numbers makes it difficult to apply the tools of linear algebra directly to the analysis of $\varepsilon$-uniformly convergent schemes (see, for example, Remark 2 to Theorem 3.2). To analyze the disturbed discrete 
problems, it is effective to use difference operators that are consistent with the original (subject to disturbance) operators with respect to the disturbances (see, for example, Section 4 and what follows).

We obtain estimates for the disturbance of the numerical solution depending on in what subdomain (in the boundary layer or outside it) the data of the boundary value problem are subjected to perturbation (see Theorem 4.1 for the base scheme and Theorem 6.1 for the decomposition scheme).

Thus, the $\varepsilon$-uniform sensitivity of the numerical solutions (to the perturbation in the data of the differential problem) in the layer and outside it is identical and the same as for regular problems in the case of uniform meshes. For small values of the parameter $\varepsilon$ the sensitivity to the perturbation of the right-hand side (in absolute disturbances) is reduced in the boundary layer. The sensitivity to the perturbation of the coefficient multiplying the highest derivative in the differential equation is reduced outside the layer (see, for example, estimate (4.19)).

It turns out that the difference scheme on a piecewise uniform mesh from [4] is well conditioned $\varepsilon$-uniformly (with the $\varepsilon$-uniform estimate for the condition number of the kind (4.20), which is the same estimate as for regular problems) if to consider the right-hand side of the discrete equation in the maximum norm weighted over the subdomains, that is, with a weight multiplier $\varepsilon \ln N$ for $\varepsilon=\mathcal{O}\left(\ln ^{-1} N\right)$ in a neighbourhood of the boundary layer (see Remark 5 in Section 4). Since the estimate for the disturbance of the numerical solution depending on the perturbation in the data of the discrete problem remains valid, then the transformation to such a weighted norm cannot be considered as preconditioning of the special difference scheme. The estimates obtained for deviation of the disturbed solutions from the solution of the boundary value problem (see Remark 7 in Section 4) allows one to choose appropriate accuracy for calculating the coefficients of the difference scheme (and hence the accuracy of the solver) on different parts of the grid domain, and also the number of mesh points which ensures the required accuracy of the numerical solution.

The question of ill "classical conditioning of schemes" on strongly nonuniform meshes has arisen in the literature; we mention [8] in which the technique of "classical preconditioning" for special difference schemes was discussed. The disturbance of solutions of $\varepsilon$-uniformly convergent difference schemes for singularly perturbed boundary value problems of the type $(2.1),(2.2)$ under perturbation of the data was considered in [12].

In the case of domain decomposition methods for singularly perturbed problems, the disturbance of solutions of $\varepsilon$-uniformly convergent schemes due to the perturbation in the data of the differential and discrete problems, and also conditioning of decomposition schemes were not considered previously.

About the contents. Problem formulation and motivation of research are given in Section 2. The estimates for the solutions of discrete problems in the case of uniform and close to uniform meshes are considered in Section 3; the estimates for the solutions of $\varepsilon$-uniformly convergent schemes on special fitted meshes condensing in the boundary layer are discussed in Section 3. In Sections 4 and 5, 6 we study the disturbances of the solutions of respectively the classical base scheme (problem (2.4)) and the decomposition scheme (problem (2.6)); the problems are considered on special meshes. In Sections 4, 5 we study admissible perturbations of the data of the boundary value problem under which the properties of the problems subject to disturbance remains valid. The estimates for the condition numbers of the schemes are considered in Sections 4, 6 . 


\section{Problem formulation. The aim of research}

We consider problems which arise in the numerical solution of singular perturbed equations with convective terms at the following stages: (a) the construction of $\varepsilon$-uniformly convergent finite difference schemes, i.e. the base scheme and the domain decomposition method; (b) the determination of conditions for perturbations in both the data of the boundary value problem and the data of the difference schemes under which the disturbance of a numerical solution tends to zero (as the number of mesh nodes grows) $\varepsilon$-uniformly.

1. For simplicity, we consider a singularly perturbed ordinary differential equation ${ }^{1}$

$$
\begin{aligned}
L_{(2.1)} u(x) & \equiv\left\{\varepsilon a(x) \frac{d^{2}}{d x^{2}}+b(x) \frac{d}{d x}\right\} u(x)=f(x), \quad x \in D, \\
u(x) & =\varphi(x), \quad x \in \Gamma .
\end{aligned}
$$

Here

$$
D=(0, d),
$$

$\Gamma=\bar{D} \backslash D$, the coefficients $a(x), b(x)$ and the right-hand side $f(x)$ are sufficiently smooth functions on $\bar{D}$, moreover, $a(x) \geq a_{0}, b(x) \geq b_{0}, x \in \bar{D}, a_{0}, b_{0}>0$; the parameter $\varepsilon$ takes arbitrary values from the half-open interval $(0,1]$.

We approximate problem $(2.1),(2.2)$ by a classical difference scheme [7]. To this end, on $\bar{D}$ we introduce the mesh

$$
\bar{D}_{h}=\bar{\omega}_{1},
$$

where $\bar{\omega}_{1}$ is a mesh with any distribution of its nodes satisfying only the condition ${ }^{2} h \leq M N^{-1}$; $h$ is the maximal stepsize of the mesh $\bar{\omega}_{1}, h=\max _{i} h_{i}, h^{i}=x^{i+1}-x^{i}, x^{i}, x^{i+1} \in \bar{\omega}_{1}, x^{0}=0$, $x^{N}=d, N+1$ is the number of nodes in the mesh $\bar{\omega}_{1}$. On this mesh, problem (2.1) is approximated by the difference (base) scheme

$$
\begin{aligned}
\Lambda z(x) & \equiv\left\{\varepsilon a(x) \delta_{\bar{x} \widehat{x}}+b(x) \delta_{x}\right\} z(x)=f(x), \quad x \in D_{h}, \\
z(x) & =\varphi(x), \quad x \in \Gamma_{h} .
\end{aligned}
$$

where the second order centered difference operator $\delta_{\bar{x} \widehat{x}}$ is defined by $\delta_{\bar{x} \widehat{x}} z\left(x^{i}\right)=$ $2\left(h^{i-1}+h^{i}\right)^{-1} \times\left(\delta_{x} z\left(x^{i}\right)-\delta_{\bar{x}} z\left(x^{i}\right)\right), \quad \delta_{x}$ and $\delta_{\bar{x}}$ are the forward and backward difference operators, cf. [7].

The following discrete maximum principle is valid for scheme (2.4), (2.3).

Lemma 2.1. Let the function $z(x), x \in \bar{D}_{h(2.3)}$ satisfy the relations $\Lambda_{(2.4)} z(x) \leq 0, x \in D_{h}$, $z(x) \geq 0, x \in \Gamma_{h}$. Then $z(x) \geq 0, x \in \bar{D}_{h}$.

2. In this subsection we describe a discrete Schwarz method for problem (2.4), (2.3) in the case of two overlapping subdomains of $\bar{D}$

$$
\bar{D}=\bar{D}^{(1)} \cup \bar{D}^{(2)}, \bar{D}^{(1)} \cap \bar{D}^{(2)} \neq \emptyset, \quad D^{(1)}=\left(0, d^{1}\right),
$$

${ }^{1}$ Throughout the paper, the notation $L_{(j . k)}\left(M_{(j . k)}, G_{h(j . k)}\right)$ means that these operators (constants, grids) are introduced in equation $(j . k)$.

${ }^{2}$ Here and below $M, M_{i}$ (or $m$ ) denote sufficiently large (small) positive constants which do not depend on $\varepsilon$ and on the discretization parameters. 


$$
D^{(2)}=\left(d^{2}, d\right), \quad d^{1}-d^{2}=l>0
$$

with interface boundaries $\gamma^{r}=\left\{x=d^{r}\right\}, r=1,2$. On the sets $\bar{D}^{(r)}$ we introduce the meshes

$$
\bar{D}_{h}^{(r)}=\bar{D}^{(r)} \cap \bar{D}_{h}, \quad r=1,2 ;
$$

assume that the boundaries $\gamma^{r}$ belong to the mesh $\bar{D}_{h} ; N^{(r)}+1$ and $N^{\gamma}+1$ are the number of nodes in the mesh $\bar{D}_{h}^{(r)}$ and on the set $\left[d^{2}, d^{1}\right]=\bar{D}^{(1)} \cap \bar{D}^{(2)}$, respectively.

Let some function $z^{0}(x)=u^{0}(x), x \in \bar{D}_{h}$ be given and suppose that the functions $z^{1}(x), \ldots$, $z^{n-1}(x), x \in \bar{D}_{h}$ have been already constructed, where $z^{i}(x)=\varphi(x), x \in \Gamma_{h}, i=1, \ldots, n-1$. We construct the function $z^{n}(x)$. At first we find the functions $z^{n(r)}(x), x \in \bar{D}_{h}^{(r)}, r=1,2$, by solving the problems

$$
\begin{aligned}
\Lambda z^{n(2)}(x) & =f(x), \quad x \in D_{h}^{(2)} \\
z^{n(2)}(x) & = \begin{cases}\varphi(x), & x \in \Gamma_{h}^{(2)} \cap \Gamma \\
z^{n-1}(x), & x \in \gamma_{h}^{2}=\left\{\Gamma_{h}^{(2)} \backslash \Gamma\right\}\end{cases} \\
\Lambda^{n(1)} z(x) & =f(x), \quad x \in D_{h}^{(1)}, \\
z^{n(1)}(x) & = \begin{cases}\varphi(x), & x \in \Gamma_{h}^{(1)} \cap \Gamma \\
z^{n(2)}(x), & x \in \gamma_{h}^{1}=\left\{\Gamma_{h}^{(1)} \backslash \Gamma\right\} .\end{cases}
\end{aligned}
$$

Then we assume

$$
z^{n}(x)= \begin{cases}z^{n(1)}(x), & x \in \bar{D}_{h}^{(1)} \\ z^{n(2)}(x), & x \in \bar{D}_{h} \backslash \bar{D}^{(1)} ; x \in \bar{D}_{h}\end{cases}
$$

we call the function $z^{n}(x), x \in \bar{D}_{h}, n=1,2, \ldots$, the solution of difference scheme (2.6), (2.5), i.e., the iterative discrete Schwarz method (or the iterative difference scheme of the domain decomposition method).

For $n \rightarrow \infty$ the solution of problem (2.6), (2.5), (2.3) converges to the stationary solution, which is the solution of the problem

$$
\begin{aligned}
& \Lambda z^{(r)}(x)=f(x), \quad x \in D_{h}^{(r)}, \\
& z^{(r)}(x)= \begin{cases}\varphi(x), & x \in \Gamma_{h}, \\
z^{(3-r)}(x), & x \in \gamma_{h}^{r} ; \quad r=1,2 .\end{cases}
\end{aligned}
$$

Note that $z^{(r)}(x)=z(x), x \in \bar{D}_{h}^{(r)}$, where $z(x)$ is the solution of problem $(2.4),(2.3), z^{(r)}(x)$, $x \in \bar{D}_{h}^{(r)}, r=1,2$ are components of the solution for problem (2.7), (2.3), i.e., the difference (non-iterative) scheme of the overlapping domain decomposition method.

For the difference schemes (2.6), (2.5) and (2.7), (2.5), (2.3) the maximum principle is valid.

Lemma 2.2. Let the functions $z^{n(r)}(x), x \in \bar{D}_{h}^{(r)}, r=1,2, n=1,2, \ldots$ satisfy the relations $\Lambda_{(2.4)} z^{n(r)}(x) \leq 0, x \in D_{h}^{(r)} ; z^{1(2)}(x) \geq 0, x \in \bar{D}_{h}^{(2)} ; z^{n(r)}(x) \geq 0, x \in \Gamma_{h} ; z^{n(1)}(x) \geq z^{n(2)}(x)$, $x \in \gamma_{h}^{1} ; z^{n(2)}(x) \geq z^{n-1(1)}(x), x \in \gamma_{h}^{2}$, where $z^{n-1(1)}(x)=0$ for $n=1$. Then $z^{n}(x) \geq 0$, $x \in \bar{D}_{h}, n=1,2, \ldots$ 
Lemma 2.3. Let the functions $z^{(r)}(x), x \in \bar{D}_{h}^{(r)}, r=1,2$ satisfy the relations $\Lambda_{(2.4)} z^{(r)} \leq 0$, $x \in D_{h}^{(r)} ; z^{(r)}(x) \geq 0, x \in \Gamma_{h} ; z^{(r)}(x) \geq z^{(3-r)}(x), x \in \gamma_{h}^{r}, r=1,2$. Then $z^{(r)}(x) \geq 0, x \in \bar{D}_{h}^{(r)}$, $r=1,2$.

3. Let us state the aim of research. An algebraic approach (see, for example, $[10,11]$ ) is very attractive for the study of approximations to problem (2.1), (2.2) In the case of uniform meshes the discrete problems (2.4), (2.3) and (2.7), (2.3) are well conditioned $\varepsilon$-uniformly. Moreover, solutions of the iterative method (2.6), (2.5) provided that

$$
l \geq m \varepsilon+N^{-1}
$$

which is not a so restrictive condition (see (3.13a)) for the width of the overlapping region, converge $\varepsilon$-uniformly (in the maximum norm) to the solution of the "stationary" discrete problem (2.7), (2.3) (and also to the solution of the base scheme (2.4), (2.3)) for $n \rightarrow \infty$. That is, the rate of convergence of the functions $z^{n}(x)$ to $z(x)$ does not depend on the value of the parameter $\varepsilon$ (see Theorem 3.1; recall that $l$ is the width of the overlapping region, $n$ is the number of an iterate). However, the discrete solutions of problem (2.4), (2.3) converge (on uniform meshes) to the solution of problem (2.1), (2.2) in the maximum norm only under the condition

$$
N^{-1} \ll \varepsilon,
$$

which is too restrictive for singularly perturbed problems.

That is why, in order to provide $\varepsilon$-uniform convergence of the solutions of scheme (2.4), (2.3), we use fitted meshes which are a priori adapted to the boundary layer. To solve problem (2.1), (2.2) we use a special piecewise uniform mesh, which is the simplest of meshes (condensed in a neighbourhood of the boundary layer) on which the solution of the difference scheme (2.4) converges (in the maximum norm) to the solution of the boundary value problem $\varepsilon$-uniformly. The condition numbers for the difference operator $\Lambda_{(2.4)}$ on such meshes are not bounded $\varepsilon$ uniformly (see Theorem 3.2). For regular problems large condition numbers lead, in general, to an increased sensitivity of numerical solutions to perturbations of the data (see, e.g., [10, 11]).

Therefore, it is very urgent to study how perturbations in the data of both boundary value problems and discrete problems influence disturbances of numerical solutions (and also errors of numerical decomposition methods).

Our aim is to derive estimates for disturbances of the numerical solutions of problem (2.1), (2.2), in particular, for the discrete Schwarz method, depending on the disturbances of the data of both the boundary value problem and its mesh approximations in the case of schemes that converge $\varepsilon$-uniformly in the maximum norm. In this paper the study is confined to the case of perturbations in the data of the boundary value problem.

\section{Auxiliary constructions}

1. To analyze discrete problems (2.4), (2.3) and (2.6), (2.3) we will use the tools of linear algebra together with the theory of difference schemes.

We write the left-hand side of discrete equations (2.4) in the canonical form

$$
\Lambda_{(2.4)} z(x)=\sum_{j=i-1}^{i+1}(-1)^{i+1-j} q_{i j} z\left(x^{j}\right) \equiv \Lambda_{(3.1)} z(x), \quad x=x^{i} \in D_{h}
$$

where $q_{i j}$ can be written in terms of the coefficients $a(x), b(x), x \in D_{h} ; q_{i j}=q_{i j}\left(a\left(x^{i}\right), b\left(x^{i}\right)\right)$, $x^{i} \in D_{h}, j=i-1, i, i+1$. 
Then we write the difference scheme (2.4), (2.3) as a system of algebraic equations. Let an $(N+1)$-dimensional vector $y$ correspond to the $N+1$ components of $z(x), x \in \bar{D}_{h}$. After ordering of the elements $z(x), x \in \bar{D}_{h}$, we come to the matrix system

$$
A y=b
$$

Here $A$ is a tridiagonal $(N+1) \times(N+1)$-matrix $\left(a_{i j}\right)$ of elements $a_{i j}=-(-1)^{i+1-j} q_{i-1, j-1}$, $i=2,3, \ldots, N-1, j=i-1, i, i+1, a_{11}=a_{N+1, N+1}=1$, the other coefficients are zero; $b$ is an $(N+1)$-dimensional vector. The components of the vector $b$ corresponding to the nodes $x \in D_{h}$ and $x \in \Gamma_{h}$ are the values of $f(x)$ and $\varphi(x)$, respectively; let the first component of the vector $b$ correspond to the point $x^{0}=0$. The tridiagonal matrix $A$ is an $M$-matrix with the properties of nonstrict diagonal dominance and strict dominance with respect to the first and last rows. Let $y$ and $b$ be vectors from normed spaces $Y$ and $B$ endowed with the maximum vector-norm $\|\cdot\|$ (i.e., the $L_{\infty}$ norm). The notation $A \geq 0$ and $y \geq 0$ means that $a_{i j} \geq 0$ and $y_{i} \geq 0$ for all $i, j$.

The operator $A_{(3.2)}$ satisfies the monotonicity principle: the condition $A y^{1} \geq A y^{2}$ implies $y^{1} \geq y^{2}$.

2. When investigating problems (2.6), (2.5), (2.3) and (2.7), (2.5), (2.3), we will operate with suitable "extended" functions on "extended" domains. Let the set $\bar{D}^{e}$ be generated by disconnected sets $\bar{D}^{(1)}$ and $\bar{D}^{(2) *}$, where $\bar{D}^{(1)}=\bar{D}_{(2.5)}^{(1)}, \bar{D}^{(2) *}$ is obtained by displacement of the set $\bar{D}_{(2.5)}^{(2)}$ on the distance $1+l_{(2.5)}$ to the right

$$
\bar{D}^{e}=\bar{D}^{(1)} \cup \bar{D}^{(2) *} ; \quad D^{e}=\left(0, d^{1}\right] \cup\left[d^{2 e}, d^{e}\right),
$$

here $d^{2 e}=d^{2}+1+l, d^{e}=d+1+l$. In a similar way, on $\bar{D}^{e}$ we construct the extended discrete set based on the mesh $\bar{D}_{h(2.3)}$

$$
\bar{D}_{h}^{e}=\bar{D}_{h}^{(1)} \cup \bar{D}_{h}^{(2) *}, \quad \bar{D}_{h}^{e}=\bar{D}_{h}^{e}\left(\bar{D}_{h}\right) ;
$$

$N^{e}+2$ is the number of nodes in the mesh $\bar{D}_{h}^{e}, N^{e}=N^{(1)}+N^{(2)}=N+N^{\gamma}$. Let $v(x), x \in \bar{D}_{h}$ be some function. Using the function $v(x)$, we construct the extended function $v^{e}(x), x \in \bar{D}_{h}^{e}$ by assuming

$$
v^{e}(x)=v^{e}(x ; v(\cdot))= \begin{cases}v(x), & x \in \bar{D}_{h}^{(1)}, \\ v(x-1-l), & x \in \bar{D}_{h}^{(2) *} ; x \in \bar{D}_{h}^{e} .\end{cases}
$$

We now write problem $(2.7),(2.3)$, but for the extended functions, in the canonical form

$$
\begin{aligned}
& \Lambda^{e} z^{e}(x) \equiv \sum_{\substack{j=i-1 \\
i \notin I_{1}}}^{i+1}(-1)^{i+1-j} q_{i j}^{e} z^{e}\left(x^{j}\right)+\sum_{\substack{j=j(i) \\
i \in I_{1}}}(-1)^{i+1-k} g_{i j} z^{e}\left(x^{j}\right)= \\
& \quad=\left\{\begin{array}{ll}
f^{e}\left(x^{i}\right), & i \notin I_{1} \\
0, & i \in I_{1}
\end{array}\right\} \equiv f_{1}^{e}\left(x^{i}\right), \quad x=x^{i} \in D_{h}^{e}, \quad i=1, \ldots, N^{e} .
\end{aligned}
$$

Here $I_{1}=\left\{i=N^{(1)}, N^{(1)}+1\right\} ; k=k(i, j), k(i, j=i)=i, k(i, j \neq i)=i+1 ; j(i)=i$, $N^{(1)}+N^{\gamma}+1$ for $i=N^{(1)}, j(i)=i, N^{(1)}-N^{\gamma}$ for $i=N^{(1)}+1$; the coefficients $q_{i j}^{e}$ for $i \notin I_{1}$ can be written in terms of $a(x), b(x): q_{i j}^{e}=q_{i j(3.1)}$ for $1 \leq i \leq N^{(1)}-1, q_{i j}^{e}=$ 


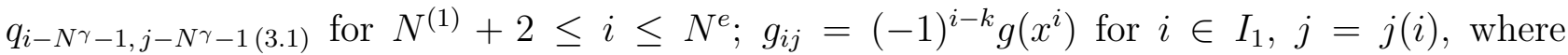
$g\left(x^{i}\right)=1 ; f^{e}(x)=f_{(3.4)}^{e}(x ; f(\cdot))$.

We consider the function $g\left(x^{i}\right), i \in I_{1}$ as well as the functions $a(x), b(x)$ to be the data of a continual problem, viz. the continual domain decomposition method on the overlapping subdomains $\bar{D}^{(1)}$ and $\bar{D}^{(2)}$ with Dirichlet interface conditions. On the boundary $\Gamma_{h}^{e}$ the function $z^{e}(x)$ takes the prescribed values

$$
z^{e}(x)=\varphi^{e}(x)=\left\{\begin{array}{ll}
\varphi(0), & i=0 \\
\varphi(d), & i=N^{e}+1
\end{array}\right\}, \quad x=x^{i} \in \Gamma_{h}^{e}, i=0, N^{e}+1 .
$$

The difference equations (3.5a) (equation (2.7)) can be also written in the following form:

$$
\begin{aligned}
\Lambda^{e} z^{e}(x) & \equiv\left\{\varepsilon a^{e}(x) \delta_{\bar{x} \widehat{x}}+b^{e}(x) \delta_{x}\right\} z^{e}(x)=f^{e}(x), \quad x=x^{i} \in D_{h}^{e}, \quad i \notin I_{1}, \\
\Lambda^{e} z^{e}(x) & \equiv g(x)\left(-z^{e}(x)+z^{e}\left(x^{j}\right)\right)=0, \quad x=x^{i} \in D_{h}^{e}, \quad i \in I_{1}, \\
& i=1, \ldots, N^{e}, \quad j=j_{(3.5 a)}(i) .
\end{aligned}
$$

We write the difference scheme (3.5), (3.3) in the algebraic form as a system of algebraic equations. Let an $\left(N^{e}+2\right)$-dimensional vector $y^{e}$ correspond to the $N^{e}+2$ components of $z^{e}(x), x \in \bar{D}_{h}^{e}$. The scheme (3.5), (3.3) results in the matrix system

$$
A^{e} y^{e}=b^{e} .
$$

Here $A^{e}$ is a tridiagonal $\left(N^{e}+2\right) \times\left(N^{e}+2\right)$-matrix with the elements $a_{i j}^{e}=-(-1)^{i+1-j} q_{i-1, j-1}^{e}$, $i-1 \notin I_{1} ; a_{i i}^{e}=-q_{i i}^{e}, i-1 \in I_{1}$, and with two additional elements

$$
a_{i j}^{e}=g_{i j}^{e}, \quad i-1 \in I_{1}, \quad j \neq i, \quad j=j(i),
$$

where $j(i)=N^{(1)}+N^{\gamma}+2$ for $i=N^{(1)}+1, j(i)=N^{(1)}-N^{\gamma}+1$ for $i=N^{(1)}+2$; $b^{e}$ is an $\left(N^{e}+2\right)$-dimensional vector (the components of the vector $b^{e}$ are the values $f^{e}\left(x^{i-1}\right)$ for $x^{i-1} \in D_{h}^{e}$ for $i \neq 1, N^{(1)}+1, N^{(1)}+2, N^{e}+2$ and the values $\varphi^{e}\left(x^{i-1}\right)$ for $\left.i=1, N^{e}+2\right)$.

3. The operator $A_{(3.6)}^{e}$ satisfies the monotonicity principle.

Using the majorant function technique (see, i.g. $[4,5,7,9]$ ) applied to the discrete problem (3.5), (3.3), we establish $\varepsilon$-uniform boundedness for the norm of the inverse matrix $\left(A^{e}\right)^{-1}$ : $\left\|\left(A^{e}\right)^{-1}\right\| \leq M$. Here $\left\|\left(A^{e}\right)^{-1}\right\|$ is the matrix norm induced by the maximum vector-norm $\|\cdot\|$.

On the meshes

$$
\bar{D}_{h}=\bar{\omega}_{1}
$$

where $\bar{\omega}_{1}=\bar{\omega}_{1(2.3)}$, whose nodes satisfy the condition $\min _{i} h^{i} \geq m N^{-1}$, in particular, on the uniform mesh

$$
\bar{D}_{h}=\bar{\omega}_{1}
$$

we have: $\|A\|,\left\|\left(A^{e}\right)^{-1}\right\| \leq M N^{2}$. Thus, for the condition number of the matrices $A_{(3.2)}$ and $A_{(3.6)}^{e}$ in the case of meshes $D_{h(3.7)}$ we obtain the estimates

$$
\begin{aligned}
& æ(A)=æ\left(A ; \bar{D}_{h(3.7)}\right) \leq M N^{2}, \\
& æ\left(A^{e}\right)=æ\left(A^{e} ; \bar{D}_{h}^{e}\left(\bar{D}_{h(3.7)}\right)\right) \leq M N^{2} .
\end{aligned}
$$


As $n \rightarrow \infty$ (for fixed $N, \varepsilon$ ) the solution of the iterative process (2.6) converges to the solution of the stationary problem (2.7) (or, shortly, the iterative process (2.6) converges) in the maximum norm at a rate of the geometric progression, that is,

$$
\left|z(x)-z^{n}(x)\right| \leq M q^{n}, \quad x \in \bar{D}_{h}
$$

In the case of mesh (3.8) we obtain the estimate

$$
q \leq\left(1+m \varepsilon^{-1} N^{-1}\right)^{-l N^{-1}},
$$

where $m$ is any number from the interval $\left(0, m_{0}\right], m_{0}=\min _{\bar{D}}\left[a^{-1}(x) b(x)\right], l=l_{(2.5)}$; the value of $q$ depends essentially on $\varepsilon, N, l$. A similar estimate for $q$ is valid on the mesh (3.7).

Definition. Let $\beta$ be some quantity such that $\beta=\beta(\alpha, \gamma)$, where $\alpha \rightarrow 0, \gamma \rightarrow \infty$, and assume that $\beta$ satisfies the estimate $|\beta| \leq \mu(\alpha, \gamma)$. We say that this estimate is unimprovable with respect to the values of $\alpha, \gamma$ if the estimate $|\beta| \leq \mu_{1}(\alpha, \gamma)$ is, in general, incorrect for $\mu_{1}(\alpha, \gamma)=o(\mu(\alpha, \gamma))$

The estimate (3.12) for $q$ is unimprovable with respect to the values of $N, \varepsilon, l N$.

The discrete solution $z^{n}(x)$ of the decomposition scheme (2.6), (3.8), generally speaking, does not converge for $N, n \rightarrow \infty$ (for example, for $n=N$ in the case $l=N^{-1}, \varepsilon=1$ ).

The condition

$$
l \geq m \varepsilon+N^{-1}
$$

is necessary and sufficient for the following estimate to be satisfied on the meshes $(3.7),(3.8)$ :

$$
q \leq 1-m
$$

For the solutions of problems (2.4), (3.8) and (2.6), (3.8) we have the (unimprovable) estimates

$$
\begin{aligned}
& |u(x)-z(x)| \leq M N^{-1}\left(\varepsilon+N^{-1}\right)^{-1}, \quad x \in \bar{D}_{h} ; \\
& \left|u(x)-z^{n}(x)\right| \leq M\left[N^{-1}\left(\varepsilon+N^{-1}\right)^{-1}+q^{n}\right], \quad x \in \bar{D}_{h} .
\end{aligned}
$$

The condition

$$
N^{-1}=o(\varepsilon)
$$

is necessary and sufficient for the convergence of the solutions of problems (2.4), (3.8) (for $N \rightarrow \infty$ ) and (2.6), (3.8) (for $N, n \rightarrow \infty$ ) to the solution of problem (2.1), (2.2).

The following theorem is valid.

Theorem 3.1. The condition numbers of the matrices $A_{(3.2)}, A_{(3.6)}^{e}$ are bounded $\varepsilon$-uniformly on the meshes (3.7); $(A)$, $\left(A^{e}\right)$ satisfy the estimates (3.9), (3.10). In the case of meshes (3.7), (3.8) the condition (3.13a) is necessary and sufficient for the $(\varepsilon, N)$-uniform convergence (as $n \rightarrow \infty$ ) of the solutions of the iterative method (2.6) to the solution of problem (2.7); for the iterative solutions the estimates (3.11), (3.12) (on the mesh (3.8)) and (3.13) hold. The solutions of the iterative method (2.6) (on the meshes (3.7), (3.8)) do not converge (for $N$, $n \rightarrow \infty)$ to the solution of problem (2.1), (2.2) for fixed values of the parameter $\varepsilon$; the condition (3.15) is necessary and, under the additional condition $a, b, f \in C^{2}(\bar{D})$, also sufficient for the convergence of the numerical solutions to the solution of problem (2.1), (2.2); the solutions of problems (2.4) and (2.6) on the mesh (3.8) satisfy the estimate (3.14). 
Remark 1. The condition numbers of the matrices $A, A^{e}$ in the case of meshes (3.7), (3.8) satisfy the following unimprovable with respect to $\varepsilon, N$ estimate

$$
æ(A), æ\left(A^{e}\right) \leq M N(1+\varepsilon N) .
$$

From this estimate and condition (3.15) it follows that the condition numbers of the matrices $A, A^{e}$ in the case of a scheme convergent on the meshes (3.7) or (3.8) grow without bound as $\varepsilon \rightarrow 0$; precisely, the following (unimprovable with respect to $\varepsilon, N$ ) lower bounds hold for the condition numbers of the matrices $A, A^{e}$ :

$$
æ(A), æ\left(A^{e}\right) \gg \varepsilon^{-1} .
$$

4. In this subsection we construct special fitted meshes which are condensing in the boundary layer region. The difference scheme (2.4) on such schemes does converge $\varepsilon$-uniformly.

On the set $\bar{D}$ we introduce the mesh

$$
\bar{D}_{h}^{*}=\bar{D}_{h}^{S}=\bar{\omega}_{1}^{S},
$$

where $\bar{\omega}_{1}^{S}=\bar{\omega}_{1}^{S}(\sigma)$ is a piecewise uniform mesh, uniform on the segments $[0, \sigma]$ and $[\sigma, d]$, $h^{(1)}$ and $h^{(2)}$ are its step-sizes on $[0, \sigma]$ and $[\sigma, d]$, respectively, defined by $h^{(1)}=2 \sigma N^{-1}$ and $h^{(2)}=2(d-\sigma) N^{-1}$,

$$
\sigma=\sigma(\varepsilon, N)=\min \left[2^{-1} d, m^{-1} \varepsilon \ln N\right],
$$

$m$ is an arbitrary number from the interval $\left(0, m^{0}\right), m^{0}=\min _{\bar{D}}\left[a^{-1}(x) b(x)\right]$.

For the quantities $\|A\|,\left\|A^{e}\right\|, æ(A), æ\left(A^{e}\right)$ on the mesh $\bar{D}_{h(3.16)}$ we have the estimate

$$
\begin{gathered}
\left\|A\left(\bar{D}_{h(3.16)}\right)\right\|,\left\|A^{e}\left(\bar{D}_{h(3.16)}\right)\right\|, æ\left(A ; \bar{D}_{h(3.16)}\right), æ\left(A^{e} ; \bar{D}_{h(3.16)}\right) \leq \\
\leq M \varepsilon^{-1} N^{2}\left(\varepsilon+\ln ^{-1} N\right)^{2}
\end{gathered}
$$

which is unimprovable with respect to $\varepsilon, N$.

Theorem 3.2. In the case of the difference scheme (2.4), (3.16) the matrices $A\left(\bar{D}_{h(3.16)}\right)$, $A^{e}\left(\bar{D}_{h(3.16)}\right)$ and the condition numbers $æ\left(A ; \bar{D}_{h(3.16)}\right)$, $\left(A^{e} ; \bar{D}_{h(3.16)}\right)$ satisfy the unimprovable estimate (3.17).

Remark 2. In the case of $\varepsilon$-uniformly convergent schemes on piecewise uniform meshes the coefficients of the operator $\Lambda_{(3.1)}$ (the elements of the matrices $A_{(3.2)}, A_{(3.6)}^{e}$ ) and also the numbers $æ(A), æ\left(A^{e}\right)$ grow without bound for $\varepsilon \rightarrow 0$. Therefore, the direct use of $æ(A), æ\left(A^{e}\right)$ in the analysis of the $\varepsilon$-uniform stability of numerical solutions does not allow us to obtain $\varepsilon$-uniform estimates for the solution disturbances with respect to disturbances of the data of problem (2.4), (3.16) (problems (3.2), (3.16) and (3.6), (3.16)).

5. When analyzing the iterative method (2.6), (3.16), for simplicity we assume the following condition to be satisfied:

$$
\text { either } \sigma \leq d^{2}, \text { or } \sigma \geq d^{1}, \quad \sigma=\sigma_{(3.16)}
$$


which ensures the uniformity of the mesh (3.16) on the overlapping region $\bar{D}^{(1)} \cap \bar{D}^{(2)}$.

In this case, for the solutions of iterative process (2.6), (3.16) we obtain the estimate

$$
\left|z(x)-z^{n}(x)\right| \leq M q^{n}, \quad x \in \bar{D}_{h},
$$

moreover, for the value $q$ we have

$$
q \leq \begin{cases}\left(1+m_{1} \varepsilon^{-1} N^{-1}\right)^{-l N}, & \sigma \leq d^{2} \\ \exp \left(-m_{2} \varepsilon^{-1} l\right), & \sigma \geq d^{1}\end{cases}
$$

where $m_{1}=2^{-1} m_{2}, m_{2}=m_{(3.16)}, l=l_{(2.5)}$; the estimate is unimprovable with respect to $N, \varepsilon, l$.

Note that $q=q(N, \varepsilon, l)$, depending on the parameters, can be arbitrarily close to 1 . However, $q$ is bounded $(\varepsilon, l)$-uniformly; we have the $(\varepsilon, l)$-uniform estimate

$$
q \leq 1-m N^{-1}
$$

which is unimprovable with respect to the value of $N$; generally speaking, the solutions of scheme (2.6), (3.16) do not converge to the solution of scheme (3.6), (3.16) for $N, n \rightarrow \infty$.

The condition

$$
l \geq \begin{cases}m\left(\varepsilon+N^{-1}\right), & \sigma \leq d^{2}, \\ m \varepsilon, & \sigma \geq d^{1},\end{cases}
$$

which is equivalent to the condition

$$
l \geq m \varepsilon
$$

is necessary and sufficient for the following estimate to be satisfied on the mesh (3.16):

$$
q \leq 1-m
$$

The solutions of problem (2.4), (3.16) and of the iterative problem (2.6), (3.16) satisfy the (unimprovable) estimates

$$
\begin{aligned}
& |u(x)-z(x)| \leq M N^{-1} \ln N, \quad x \in \bar{D}_{h} ; \\
& \left|u(x)-z^{n}(x)\right| \leq M\left[N^{-1} \ln N+q^{n}\right], \quad x \in \bar{D}_{h} .
\end{aligned}
$$

The solutions of problems (2.4), (3.16) (for $N \rightarrow \infty$ ) and (2.6), (3.16), (3.21a) (for $N, n \rightarrow$ $\infty)$ converge to the solution of problem (2.1), (2.2) $\varepsilon$-uniformly. However, if condition (3.21a) fails, the solution of problem (2.6), (3.16), in general, does not converge to the solution of problem (2.1), (2.2) for $N, n \rightarrow \infty$, i.e., under the violation of condition (3.21a) the convergence is not unconditional (with respect to $N, n$ ).

Theorem 3.3. The condition (3.21a) is necessary and, if $a, b, f \in C^{2}(\bar{D})$, also sufficient in order that the solutions of the decomposition scheme (2.6), (3.16) for $N, n \rightarrow \infty$ converge to the solution of the boundary value problem (2.1), (2.2) $\varepsilon$-uniformly. For the solutions of problems (2.4), (3.16) and (2.6), (3.16), and also for the value of $q$ the estimates (3.19), (3.22) and (3.21) are valid. 


\section{Grid approximations of the boundary value problem $(2.1), 2.2)$ in the case of the disturbance of its data}

1. For the analysis of problem (2.4), (3.16) it is convenient to consider the set $D_{h}$ as a composition of several subsets. Let

$$
D_{h}=\bigcup D_{h}^{k}, \quad k=1,2,3,
$$

where $D_{h}^{1}=(0, \sigma) \cap D_{h}, D_{h}^{2}=\{x=\sigma\} \cap D_{h}, \quad D_{h}^{3}=(\sigma, d) \cap D_{h}, \quad \sigma \in D_{h}$. For piecewise constant functions $v(x), x \in D_{h}$, which are constant on the sets $D_{h(4.1)}^{k}$, we use the "vector" notation

$$
v(x)=\left\{v_{1}, v_{2}, v_{3}\right\}, \quad x \in D_{h}, \quad v_{k}=v(x), \quad x \in D_{h}^{k}, \quad k=1,2,3 .
$$

The coefficients $q_{i j(3.1)}$ of the difference scheme depend on the functions $a(x), b(x), x \in \bar{D}$. Let the coefficients $q_{i j}, i=1, \ldots, N-1, j=i-1, i, i+1$ and the functions $f(x), x \in D_{h}, \varphi(x)$, $x \in \Gamma_{h}$ in equations (2.4), (3.1) take some disturbed values $q_{i j}^{*}, f^{*}(x), \varphi^{*}(x)$ respectively. Then the difference scheme (2.4), (3.16) and problem (3.2) transform respectively into the perturbed difference scheme

$$
\begin{aligned}
\Lambda^{*} z^{*}(x) & \equiv \sum_{j=i-1}^{i+1}(-1)^{i+1-j} q_{i j}^{*} z^{*}\left(x^{j}\right)=f^{*}(x), \quad x=x^{i} \in D_{h}, \\
z^{*}(x) & =\varphi^{*}(x), \quad x \in \Gamma_{h}
\end{aligned}
$$

and into the perturbed problem

$$
A^{*} y^{*}=b^{*} \text {. }
$$

For further constructions it will be convenient to group together the terms of the difference scheme (4.2), (3.16) and to write the operator $\Lambda^{*}$ in the following form, using the unknown function and its differences $\delta_{\bar{x} \widehat{x}}, \delta_{x}$ :

$$
\begin{array}{r}
\Lambda_{(4.2)}^{*} z^{*}(x)=\left\{\varepsilon \widetilde{a}(x) \delta_{\bar{x} \widehat{x}}+\widetilde{b}(x) \delta_{x}+\widetilde{c}(x)\right\} z^{*}(x) \equiv \\
\equiv\left(\widetilde{\Lambda}_{(4.4)}^{p}+\widetilde{c}(x)\right) z^{*}(x) \equiv \widetilde{\Lambda}_{(4.4)} z^{*}(x), \quad x \in D_{h} .
\end{array}
$$

We confine our consideration to the case when the perturbed coefficients $q_{i j}^{*}$ are generated by the perturbed functions $a^{*}(x), b^{*}(x)$, which correspond to the functions $a(x), b(x), x \in D_{h}$, and so there are no other disturbances of $q_{i j}$, except these ones.

In the case of the disturbances of scheme (2.4), (3.16) which are caused by disturbances of the data of problem (2.1), (2.2), the quantities $q_{i j}^{*}$, i.e., the perturbed values of the quantities $q_{i j}=q_{i j}\left(a\left(x^{i}\right), b\left(x^{i}\right)\right)$, are defined by $a^{*}\left(x^{i}\right), b^{*}\left(x^{i}\right)$ (approximations to $\left.a\left(x^{i}\right), b\left(x^{i}\right)\right)$ taking the values $a_{j}^{*}\left(x^{i}\right), b_{j}^{*}\left(x^{i}\right), j=i-1, i, i+1$ :

$$
q_{i j}^{*}=q_{i j}\left(a_{j}^{*}\left(x^{i}\right), b_{j}^{*}\left(x^{i}\right)\right) ;
$$

it is not assumed that $a_{j}^{*}\left(x^{i}\right)=a_{k}^{*}\left(x^{i}\right), b_{j}^{*}\left(x^{i}\right)=b_{k}^{*}\left(x^{i}\right)$ for $j \neq k$; in general,

$$
\begin{aligned}
a_{j}^{*}\left(x^{i}\right) & \neq a_{k}^{*}\left(x^{i}\right), \quad b_{j}^{*}\left(x^{i}\right) \neq b_{k}^{*}\left(x^{i}\right), \quad x^{i} \in D_{h}, \\
j & \neq k, \quad j, k=i-1, i, i+1 .
\end{aligned}
$$


For the disturbances we use the notations

$$
\begin{aligned}
& z^{\delta}(x)=z^{*}(x)-z(x), \quad q_{i j}^{\delta}=q_{i j}^{*}-q_{i j}, \\
& a^{\delta}(x)=a^{*}(x)-a(x), \ldots, \quad y^{\delta}=y^{*}-y .
\end{aligned}
$$

It is required to find the conditions for the disturbances of the data of boundary value problem (2.1), (2.2) under which the solution of the difference scheme (4.2), (3.16) (problem (4.3)) exists, is $\varepsilon$-uniformly bounded and converges (for $N \rightarrow \infty$ ) to the solution of the difference scheme (2.4), (3.16) (problem (3.2)) $\varepsilon$-uniformly. From the $\varepsilon$-uniform convergence of $z^{*}(x), x \in \bar{D}_{h}$ to $z(x)$ :

$$
\left|z^{*}(x)-z(x)\right| \leq \lambda(N), \quad x \in \bar{D}_{h}
$$

it follows that the solutions of the perturbed difference scheme (4.2), (3.16) converge $\varepsilon$-uniformly to the solution of the boundary value problem (2.1), (2.2). So, in the case of estimate (3.22a) we have

$$
\left|u(x)-z^{*}(x)\right| \leq M N^{-1} \ln N+\lambda(N), \quad x \in \bar{D}_{h}
$$

2. We now give some estimates for the disturbances of the data of scheme (4.2), (3.16), which are generated by the disturbances of the data of boundary value problem (2.1), (2.2).

2.1. For the coefficients of the operators $\widetilde{\Lambda}_{(4.4)}$ and $\Lambda_{(4.2)}^{*}$ we obtain the estimates

$$
\begin{aligned}
|\widetilde{a}(x)-a(x)| & \leq M_{1}\left|a^{\delta}(x)\right|, \\
|\widetilde{b}(x)-b(x)| & \leq M_{2}\left[\eta_{1}(x)\left|a^{\delta}(x)\right|+\left|b^{\delta}(x)\right|\right], \\
|\widetilde{c}(x)| & \leq M_{3}\left[\eta_{2}(x)\left|a^{\delta}(x)\right|+\eta_{3}(x)\left|b^{\delta}(x)\right|\right], \quad x \in D_{h} ; \\
\left|q_{i j}^{\delta}\right| & \leq M_{4}\left[\eta_{2}\left(x^{i}\right)\left|a^{\delta}\left(x^{i}\right)\right|+\eta_{3}\left(x^{i}\right)\left|b^{\delta}\left(x^{i}\right)\right|\right], \quad x^{i} \in D_{h} ; \\
\eta_{1}(x) & =\left\{N\left(\varepsilon+\ln ^{-1} N\right), \varepsilon N, \varepsilon N\right\}, \\
\eta_{2}(x) & =\left\{\varepsilon^{-1} N^{2}\left(\varepsilon+\ln ^{-1} N\right)^{2}, N^{2}\left(\varepsilon+\ln ^{-1} N\right), \varepsilon N^{2}\right\}, \\
\eta_{3}(x) & =\left\{\varepsilon^{-1} N\left(\varepsilon+\ln ^{-1} N\right), N, N\right\} ;
\end{aligned}
$$

here and in what follows $\eta_{k}(x)=\eta_{k}(x ; \varepsilon, N)$ are piecewise constant functions in the vector notation (4.1b); the disturbances $a^{\delta}(x), b^{\delta}(x), q_{i j}^{\delta}$ are defined by (4.6). The estimates (4.7) are unimprovable with respect to $a^{\delta}(x), b^{\delta}(x), \varepsilon, N$.

2.2. In that case when the disturbances satisfy the conditions

$$
\begin{gathered}
\left|a^{\delta}(x)\right| \leq m_{1} \eta(x), \quad\left|b^{\delta}(x)\right| \leq m_{2}, \quad x \in D_{h}, \\
\eta(x)=\left\{N^{-1}\left(\varepsilon+\ln ^{-1} N\right)^{-1},(1+\varepsilon N)^{-1},(1+\varepsilon N)^{-1}\right\},
\end{gathered}
$$

for the coefficients of the operator $\widetilde{\Lambda}_{(4.4)}$ we have the estimate

$$
|\widetilde{a}(x)-a(x)|,|\widetilde{b}(x)-b(x)| \leq m, \quad x \in D_{h},
$$

where $m=m_{(4.9)}\left(m_{1(4.8)}, m_{2(4.8)}\right) \rightarrow 0$ for $m_{i(4.8)} \rightarrow 0$. Under conditions (4.8), for the function $\widetilde{c}(x)$ we have

$$
\begin{gathered}
|\widetilde{c}(x)| \leq m \eta(x), \quad x \in D_{h}, \\
\eta(x)=\left\{\varepsilon^{-1} N\left(\varepsilon+\ln ^{-1} N\right), N+N^{2} \ln ^{-1} N(1+\varepsilon N)^{-1}, N\right\},
\end{gathered}
$$


where $m=m\left(m_{(4.8)}\right)$; the estimate $(4.10)$ is unimprovable with respect to $\varepsilon, N$.

2.3. In the case of sufficiently large values of $m_{1(4.8)}, m_{2(4.8)}$ the coefficients $\widetilde{a}(x), \widetilde{b}(x)$ differ greatly from $a(x), b(x)$ and can be negative.

Definition. We say that the operator $\widetilde{\Lambda}_{(4.4)}^{p}$, i.e., the main term of the operator $\widetilde{\Lambda}_{(4.4)}$, and the operator $\Lambda_{(2.4)}$ (its main term) are consistent with respect to the disturbances $a^{\delta}(x), b^{\delta}(x)$ satisfying the conditions (4.8) (or, shortly, with respect to the disturbances $a^{\delta}(x), b^{\delta}(x)$ ) if their coefficients satisfy the estimate (4.9).

Lemma 4.1. Under the conditions (4.8) the operators $\widetilde{\Lambda}_{(4.4)}^{p}$ and $\Lambda_{(2.4)}$ are consistent with respect to the disturbances $a^{\delta}(x), b^{\delta}(x)$; for the coefficients $\widetilde{a}(x), \widetilde{b}(x)$ (the coefficient $\widetilde{c}(x)$ ) under the conditions (4.8) the estimate (4.9) (the estimate (4.10)) is valid.

Note that the function $z^{\delta}(x), x \in \bar{D}_{h}$, by virtue of formulas (2.4) and (4.2), is the solution of the difference scheme

$$
\begin{aligned}
\widetilde{\Lambda}_{(4.4)} z^{\delta}(x) & =f^{\delta}(x)-\left(\widetilde{\Lambda}_{(4.4)}-\Lambda_{(2.4)}\right) z(x), \quad x \in D_{h}, \\
z^{\delta}(x) & =\varphi^{\delta}(x), \quad x \in \Gamma_{h} ;
\end{aligned}
$$

here $z(x), x \in \bar{D}_{h}$ is the solution of problem (2.4), (3.16).

3. Before we proceed to the further study of problem (4.2), (3.16) (problem (4.11), (3.16)), we consider the simpler difference scheme

$$
\widetilde{\Lambda}_{(4.13)} \omega^{\delta}(x)=F^{\delta}(x), \quad x \in D_{h}, \quad \omega^{\delta}(x)=\varphi^{\delta}(x), \quad x \in \Gamma_{h} .
$$

Here the main part of the operator $\widetilde{\Lambda}_{(4.13)}$ in the discrete equations is the operator $\widetilde{\Lambda}_{(4.4)}^{p}$, i.e.,

$$
\begin{aligned}
& \widetilde{\Lambda}_{(4.13)} \widetilde{z}(x)=\left\{\varepsilon \widetilde{a}(x) \delta_{\widehat{x} \widehat{x}}+\widetilde{b}(x) \delta_{x}+\widetilde{c}(x)\right\} \widetilde{z}(x)= \\
& =\left(\widetilde{\Lambda}_{(4.4)}^{p}+\widetilde{c}(x)\right) \widetilde{z}(x), \quad x \in D_{h},
\end{aligned}
$$

where $\bar{D}_{h}=\bar{D}_{h(3.16)}, \widetilde{c}_{(4.13)}(x), x \in D_{h}$ as opposed to $\widetilde{c}_{(4.4)}(x)$ does not depend on the data of problem (4.2), (3.16); in general, $\widetilde{c}_{(4.13)}(x) \neq \widetilde{c}_{(4.4)}(x)$ and $\widetilde{\Lambda}_{(4.13)} \neq \widetilde{\Lambda}_{(4.4)}=\Lambda_{(4.2)}^{*}, x \in D_{h}$; $\tilde{f}(x)=f^{*}(x), x \in D_{h}, \widetilde{\varphi}(x)=\varphi^{*}(x), x \in \Gamma_{h}$.

Let the condition (4.8) hold for problem (4.12), (3.16), which ensures the proper structure of the operator $\widetilde{\Lambda}_{(4.4)}$ ), and also let the following condition be valid:

$$
\begin{aligned}
|\widetilde{c}(x)| \leq & m \eta_{(4.14)}(x), \quad x \in D_{h}, \\
\left|F^{\delta}(x)\right| \leq M \eta_{(4.14)}(x), \quad x \in D_{h}, \quad\left|\varphi^{\delta}(x)\right| \leq M, \quad x \in \Gamma_{h}, & \\
& \eta_{(4.14)}(x)=\left\{\varepsilon^{-1}\left(\varepsilon+\ln ^{-1} N\right), N, 1\right\},
\end{aligned}
$$

where $m$ is sufficiently small. Then the solution of the difference scheme (4.12), (3.16) is $(\varepsilon, N)$-uniformly bounded. The conditions (4.8), (4.14) are unimprovable with respect to $\varepsilon, N$ in order to comply with the following properties: (a) the operators $\Lambda_{(2.4)}$ and $\widetilde{\Lambda}_{(4.4)}^{p}$ are consistent with respect to the disturbances $a^{\delta}(x), b^{\delta}(x)$; (b) the solution of the difference scheme (4.12), (3.16) exists for any $\varepsilon$ and $N$; (c) the solution of this scheme is $(\varepsilon, N)$-uniformly bounded. For example, for sufficiently large $m_{i(4.8)}$ and $m_{(4.14)}$ the solution of the difference scheme, in 
general, does not exist or is not $(\varepsilon, N)$-uniformly bounded, while in the case $M_{(4.14)}=(o(1))^{-1}$ for $N \rightarrow \infty$ the solution of the difference scheme is not $(\varepsilon, N)$-uniformly bounded.

Under conditions (4.8), (4.14a) the solution of scheme (4.12), (3.16) satisfy the estimate

$$
\left|\omega^{\delta}(x)\right| \leq M\left[\max _{D_{h}}\left[\eta_{(4.14)}^{-1}(x)\left|F^{\delta}(x)\right|\right]+\max _{\Gamma_{h}}\left|\varphi^{\delta}(x)\right|\right], \quad x \in \bar{D}_{h}
$$

This estimate is unimprovable with respect to the values of $\varepsilon, N$; here $\eta_{(4.14)}(x)=$ $\eta_{(4.14)}(x ; \varepsilon, N)$, moreover, $\eta_{(4.14)}(x) \geq 1, x \in D_{h}$.

Lemma 4.2. Let the disturbances $a^{\delta}(x), b^{\delta}(x), x \in D_{h}$ of the difference scheme (4.2), (3.16) and the function $\widetilde{c}_{(4.13)}(x), x \in D_{h}$ satisfy the conditions (4.8), (4.14). Then the following statement is valid: the operators $\Lambda_{(2.4)}$ and $\widetilde{\Lambda}_{(4.4)}^{p}$ are consistent with respect to the disturbances $a^{\delta}(x), b^{\delta}(x), x \in D_{h}$, the solution of the difference scheme (4.12), (3.16) exists for any $\varepsilon$ and $N$ and is E-uniformly bounded; for this statement to be true the conditions (4.8), (4.14) are unimprovable with respect to $\varepsilon, N$. For the solutions of the difference scheme (4.12), (3.16), under the conditions (4.8), (4.14a) the estimate (4.15) holds.

Remark 3. From estimate (4.15) it follows that the identical (in module) functions $F^{\delta}(x)$ from the different subdomains $D_{h}^{k}, k=1,2,3$ contribute differently to the function $\omega^{\delta}(x)$. One can observe the essential (with respect to the values of $\varepsilon, N$ ) anisotropy (over the subdomains $\left.D_{h}^{k}\right)$ concerning the influence of the right-hand side of the difference scheme (4.12), (3.16) on its solution. The function $\omega^{\delta}(x)$ is $\varepsilon$-uniformly bounded for the functions $F^{\delta}(x)$ being not $\varepsilon$-uniformly bounded on $D_{h}^{1}$ (see condition $(4.14 \mathrm{~b})$ ).

4. In this subsection we consider the conditioning of the difference scheme (4.2), (3.16) obtained from scheme (2.4), (3.16) under disturbance of the data of the boundary value problem (2.1), (2.2). We first study the conditioning of the difference scheme (4.12), (3.16).

4.1. To estimate the solution of scheme (4.12), (3.16), it is convenient to measure the function

$$
f(x)=f\left(x ; F^{\delta}(\cdot), \varphi^{\delta}(\cdot)\right) \equiv\left\{F^{\delta}(x), \varphi^{\delta}(x)\right\}, \quad x \in \bar{D}_{h},
$$

where $f(x)=F^{\delta}(x), x \in D_{h}, f(x)=\varphi^{\delta}(x), x \in \Gamma_{h}$, i.e., the right-hand side of the discrete equations, in the special weight norm $\|\cdot\|^{S}$ :

$$
\|f(x)\|_{D^{h}}^{S}=\max \left\{\max _{D_{h}}\left[\eta_{(4.14)}^{-1}(x)\left|F^{\delta}(x)\right|\right], \max _{\Gamma_{h}}\left|\varphi^{\delta}(x)\right|\right\} .
$$

Then the estimate (4.15) takes the form

$$
\left|\omega^{\delta}(x)\right| \leq M\left\|f\left(x ; F^{\delta}(\cdot), \varphi^{\delta}(\cdot)\right)\right\|^{S}, x \in \bar{D}_{h} .
$$

Thus, the difference scheme (4.12), (3.16) (scheme (4.2), (3.16)) can be regarded as a transformation which translates the discrete space with the norm $\|\cdot\|$ (maximum grid norm) into a discrete space endowed with the special norm $\|\cdot\|_{(4.16)}^{S}$, and so the quantity $æ^{S}$ can be considered as the condition number for this transformation.

4.2. The estimate (4.15) (unimprovable with respect to $\varepsilon, N)$ and the following estimate

$$
\begin{gathered}
\max _{|\omega(\cdot)| \leq 1, D_{h}^{k}}\left|\widetilde{\Lambda}_{(4.13)} \omega(x)\right| \leq M \eta(x), \quad k=1,2,3, \quad x \in D_{h}^{k}, \\
\eta(x)=\left\{\varepsilon^{-1} N^{2}\left(\varepsilon^{2}+\ln ^{-2} N\right), N^{2}\left(\varepsilon+\ln ^{-1} N\right), N(1+\varepsilon N)\right\}
\end{gathered}
$$


imply the required estimates for the quantities $æ^{S}\left(\widetilde{\Lambda}_{(4.13)}\right)$ and $æ^{S k}\left(\widetilde{\Lambda}_{(4.13)}\right)$, where

$$
æ^{S k}\left(\widetilde{\Lambda}_{(4.13)}\right)=\max _{|\omega(\cdot)| \leq 1, D_{h}^{k}}\left[\left|\widetilde{\Lambda}_{(4.13)} \omega(x)\right| \eta_{(4.14)}^{-1}(x)\right], \quad k=1,2,3
$$

are the fractional condition numbers for problem (4.12), (3.16). We thus have the following estimates:

$$
\begin{gathered}
\mathfrak{x}^{S}\left(\widetilde{\Lambda}_{(4.13)}\right) \leq M \eta^{1} ; \quad \mathfrak{x}^{S k}\left(\widetilde{\Lambda}_{(4.13)}\right) \leq M \eta^{2}(x), \quad k=1,2,3, \quad x \in D_{h}^{k} \\
\eta^{1}=\eta^{1}(\varepsilon, N)=N^{2}\left(\varepsilon+\ln ^{-1} N\right) \leq N^{2} \\
\eta^{2}(x)=\left\{N^{2}\left(\varepsilon+\ln ^{-1} N\right), N\left(\varepsilon+\ln ^{-1} N\right), N(1+\varepsilon N)\right\} .
\end{gathered}
$$

These estimates for $æ^{S}, æ^{S k}$ are unimprovable with respect to $\varepsilon, N$.

Lemma 4.3. Let the disturbances $a^{\delta}(x), b^{\delta}(x), x \in D_{h}$ of the difference scheme (4.2), (3.16) and the function $\widetilde{c}_{(4.13)}(x), x \in D_{h}$ satisfy the conditions (4.8), (4.14a). Then the difference scheme (4.12), (3.16) is well conditioned $\varepsilon$-uniformly in the case of the special norm $\|\cdot\|_{(4.16)}^{S}$; for the condition numbers $æ^{S}\left(\widetilde{\Lambda}_{(4.13)}\right)$ and $æ^{S k}\left(\widetilde{\Lambda}_{(4.13)}\right)$ the estimates $(4.17)$ hold.

Remark 4. In the case of scheme (4.12), (3.16) a small relative disturbance of the righthand side and the boundary conditions in the special norm (i.e., the small quantity $\left\|f_{0}^{\delta}(x)\right\|^{S}$ $\left(\left\|f_{0}(x)\right\|^{S}\right)^{-1}$, where $\left.f_{0}(x)=\left\{F_{0}(x), \varphi_{0}(x)\right\}, F_{0}(x)=F_{(4.12)}^{\delta}(x), \varphi_{0}(x)=\varphi_{(4.12)}^{\delta}(x)\right)$ causes (stipulates) a small relative disturbance of the solution of the difference scheme in the maximum discrete norm (i.e., the smallness of the quantity $\left.\left\|\omega_{0}^{\delta}(x)\right\|\left(\left\|\omega_{0}(x)\right\|\right)^{-1}, \omega_{0}(x)=\omega_{(4.12)}^{\delta}(x)\right)$. Thus, the difference scheme (4.12), (3.16), in the case of the special norm, is well conditioned $\varepsilon$-uniformly; the conditioning of the difference scheme differs essentially over the subsets $D_{h}^{k}$, $k=1,2,3$.

Remark 5. Having in mind estimate (4.15), i.e., the dependence of the solution of the difference scheme (4.12), (3.16) on the right-hand side $F^{\delta}(x), x \in D_{h}$, it seems appropriate to write the difference scheme (4.12), (3.16) such that the solution of the difference scheme depends on the right-hand side "identically" on each of the subsets $D_{h}^{k}, k=1,2,3$. Multiplying the difference equations in (4.12), which correspond to $x^{i} \in D_{h}^{1}$ and $x^{i} \in D_{h}^{2}$, by the values $\varepsilon\left(\varepsilon+\ln ^{-1} N\right)^{-1}$ and $N^{-1}$ respectively, we obtain

$$
\begin{aligned}
\Lambda^{S} \omega^{\delta}(x) & \equiv\left\{\varepsilon a^{S}(x) \delta_{\bar{x} \widehat{x}}+b^{S}(x) \delta_{x}+c^{S}(x)\right\} \omega^{\delta}(x)=F^{S}(x), \quad x \in D_{h}, \\
\omega^{\delta}(x) & =\varphi^{\delta}(x), \quad x \in \Gamma_{h} .
\end{aligned}
$$

Here $v^{S}(x)=g(x) \widetilde{v}(x), \widetilde{v}(x)$ is one of the functions $\widetilde{a}(x), \widetilde{b}(x), \widetilde{c}(x), F^{\delta}(x), x \in D_{h}, g(x)=$ $\eta_{(4.14)}^{-1}(x ; \varepsilon, N)$; note that $\eta_{(4.14)}^{-1}(x ; \varepsilon, N) \approx \varepsilon \ln N$ for $x \in D_{h}^{1}, \varepsilon=\mathcal{O}\left(\ln ^{-1} N\right)$. The solution of the difference scheme (4.18), (3.16) satisfies the estimate

$$
\left|\omega^{\delta}(x)\right| \leq M \max \left\{\max _{D_{h}}\left|F^{S}(x)\right|, \max _{\Gamma_{h}}\left|\varphi^{\delta}(x)\right|\right\}, x \in \bar{D}_{h} ;
$$

for the condition number of the discrete operator $\Lambda_{(4.18)}^{S}$ we have the estimate

$$
æ\left(\Lambda_{(4.18)}^{S}\right) \leq M \eta_{(4.17)}^{1}(\varepsilon, N)
$$


5. Using the majorant function technique we estimate the solution of the difference scheme (4.11), (3.16) (the disturbance of the solution in the case of the perturbed scheme (4.2), (3.16)).

Under conditions (4.8), (4.14a) the following estimate holds for the solutions of scheme (4.11), (3.16):

$$
\begin{aligned}
& \left|z^{\delta}(x)\right| \leq M\left\{\operatorname { m a x } _ { D _ { h } } \left\{\eta _ { ( 4 . 1 4 ) } ^ { - 1 } ( x ) \left[\eta_{2(4.7)}(x)\left|a^{\delta}(x)\right|+\right.\right.\right. \\
& \left.\left.\quad+\eta_{3(4.7)}(x)\left|b^{\delta}(x)\right|\right]\right\} \max _{\bar{D}_{h}}|z(x)|+\max _{D_{h}}\left[\eta_{(4.14)}^{-1}(x)\left|f^{\delta}(x)\right|\right]+ \\
& \left.\quad+\max _{\Gamma_{h}}\left|\varphi^{\delta}(x)\right|\right\}, \quad x \in \bar{D}_{h} .
\end{aligned}
$$

For the condition numbers of the difference scheme (4.11), (3.16) (scheme (4.2), (3.16)), in the case of the special norm we have the estimates

$$
\begin{aligned}
& \mathfrak{x}^{S}\left(\Lambda_{(4.2)}^{*}\right)=\mathfrak{x}^{S}\left(\widetilde{\Lambda}_{(4.4)}\right) \leq M \eta_{(4.17)}^{1}(\varepsilon, N), \\
& æ^{S k}\left(\Lambda_{(4.2)}^{*}\right)=æ^{S k}\left(\widetilde{\Lambda}_{(4.4)}\right) \leq M \eta_{(4.17)}^{2}(x), \quad x \in D_{h}^{k}, \quad k=1,2,3 .
\end{aligned}
$$

Estimates (4.19), (4.20) are unimprovable with respect to $\varepsilon, N$. Note that the $\varepsilon$-uniform estimate for $æ^{S}\left(\Lambda_{(4.2)}^{*} ; \bar{D}_{h(3.16)}\right)$ is similar to estimate $(3.9)$, which is the estimate for $æ(A)=$ $æ\left(\Lambda_{(2.4)} ; \bar{D}_{h(3.7)}\right)$.

Under conditions (4.8), (4.14a), and also the condition

$$
\left|f^{\delta}(x)\right| \leq M \eta_{(4.14)}(x), \quad x \in D_{h}, \quad\left|\varphi^{\delta}(x)\right| \leq M, \quad x \in \Gamma_{h},
$$

the solution of the difference scheme (4.11), (3.16) is $(\varepsilon, N)$-uniformly bounded.

Theorem 4.1. Let the disturbances of the data of the boundary value problem (2.1), (2.2), which generate in turn the disturbance of the difference scheme (2.4), (3.16), satisfy the conditions (4.8), (4.14a). Then the following statements are valid: (a) the operators $\Lambda_{(2.4)}$ and $\widetilde{\Lambda}_{(4.4)}^{p}$ are consistent with respect to the disturbances $a^{\delta}(x), b^{\delta}(x), x \in D_{h}$, moreover, the solution of the difference scheme (4.2), (3.16) exists for any $\varepsilon \in(0,1]$ and $N$; for the statement (a) to be true the conditions (4.8), (4.14a) are unimprovable with respect to $\varepsilon, N$; (b) the condition numbers of the difference scheme (4.2), (3.16) and the solutions of the difference scheme (4.11), (3.16) satisfy the estimates (4.19), (4.20); these estimates are unimprovable with respect to $\varepsilon$, $N$; the condition (4.21) is necessary and sufficient for the solution of the difference scheme (4.2), (3.16) to be $(\varepsilon, N)$-uniformly bounded.

Remark 6. Let the hypothesis of Theorems 3.3, 4.1 be fulfilled. Then we have the estimate

$$
\begin{aligned}
\mid u(x) & -z^{*}(x) \mid \leq M\left\{N^{-1} \ln N+\max _{D_{h}}\left\{\eta _ { ( 4 . 1 4 ) } ^ { - 1 } ( x ) \left[\eta_{2(4.7)}(x)\left|a^{\delta}(x)\right|+\right.\right.\right. \\
& \left.\left.+\eta_{3(4.7)}(x)\left|b^{\delta}(x)\right|\right]\right\}\left[\max _{D_{h}}|f(x)|+\max _{\Gamma_{h}}|\varphi(x)|\right]+ \\
& \left.+\max _{D_{h}}\left[\eta_{(4.14)}^{-1}(x)\left|f^{\delta}(x)\right|\right]+\max _{\Gamma_{h}}\left|\varphi^{\delta}(x)\right|\right\}, \quad x \in \bar{D}_{h} .
\end{aligned}
$$


Remark 7. The estimate (4.22) allows us to choose appropriate accuracy for calculating $a(x), b(x), f(x), x \in D_{h}$ on the subdomains $D_{h}^{k}$ and the number of mesh points which ensures the required accuracy of the solution (computed on an absolutely exact computer) for the difference scheme (4.2), (3.16).

\section{Grid approximations of the domain decomposition method in the case of the disturbance of the data of the boundary value problem}

1. At first we introduce some notations. For the analysis of the decomposition scheme (3.5), (3.16) it is convenient to consider the set $D_{h}^{e}$ as a composition of several subsets. Let

$$
\widehat{D}_{h}^{e}=D_{h}^{e} \backslash \gamma_{h}^{e}, \quad \gamma_{h}^{e}=\left\{x^{i}: \quad i \in I_{1}\right\}
$$

and let

$$
D_{h}^{e}=\cup D_{h}^{k}, \quad k=1, \ldots, 5 .
$$

Here $D_{h(5.1)}^{1}$ and $D_{h(5.1)}^{3}$ are meshes with constant stepsizes equal respectively to $h^{(1)}$ and $h^{(2)}$, $h^{(i)}=h_{(3.16)}^{(i)}, D_{h}^{2}$ is the set of transition points where the mesh on $\widehat{D}_{h}^{e}$ switches from coarse to fine; $D_{h}^{4}=\left\{x^{i}: i=N^{(1)}\right\}, D_{h}^{5}=\left\{x^{i}: i=N^{(1)}+1\right\}, \gamma_{h}^{e}=D_{h}^{4} \cup D_{h}^{5}$. For piecewise constant functions $v(x), x \in D_{h}^{e}$, which are constant on the sets $D_{h(5.1)}^{k}$, we use the "vector" notation

$$
\begin{aligned}
& v(x)=\left\{v_{1}, \ldots, v_{5}\right\}=\left\{\widehat{v}, v^{\gamma}\right\}, \quad x \in D_{h}^{e} ; v^{\gamma}=\left\{v_{4}, v_{5}\right\}, \quad x \in \gamma_{h}^{e} \\
& \widehat{v}(x)=\left\{v_{1}, v_{2}, v_{3}\right\}, \quad x \in \widehat{D}_{h}^{e}, v_{k}=v(x), \quad x \in D_{h}^{k}, \quad k=1, \ldots, 5 .
\end{aligned}
$$

The coefficients $q_{i j(3.5)}^{e}, g_{i j(3.5)}$ of the difference scheme (3.5), (3.3) depend on the functions $a(x), b(x), x \in \bar{D}$, and also $g(x)_{(3.5)}$. Let the coefficients $q_{i j}^{e}, g_{i j}$ and the functions $f^{e}(x), x \in D_{h}^{e}$, $\varphi^{e}(x), x \in \Gamma_{h}^{e}$ in equations (3.5a) take disturbed values $q_{i j}^{e *}, g_{i j}^{*}, f^{e *}(x), \varphi^{e *}(x)$ respectively. Then the difference scheme (3.5), (3.3) and problem (3.6) transform respectively into the perturbed difference scheme

$$
\begin{gathered}
\Lambda^{e *} z^{e *}(x) \equiv \sum_{\substack{j=i-1 \\
i \notin I_{1}}}^{i+1}(-1)^{i+1-j} q_{i j}^{e *} z^{e *}\left(x^{j}\right)+\sum_{\substack{j=j(i) \\
i \in I_{1}}}(-1)^{i+1-k} g_{i j}^{*} z^{e *}\left(x^{j}\right)=f_{1}^{e *}\left(x^{i}\right), \\
z^{e *}(x)=\varphi^{e *}(x), \quad x \in x^{i} \in \Gamma_{h}^{e},
\end{gathered}
$$

where $k=k_{(3.5)}(i, j), j(i)=j_{(3.5)}(i)$, and into the perturbed problem

$$
A^{e *} y^{e *}=b^{e *} \text {. }
$$

We group the terms in the difference scheme (5.2), (3.16) and write the operator $\Lambda_{(5.2)}^{e *}$ in terms of the derivatives $\delta_{\bar{x} \widehat{x}}$ and $\delta_{x}$ as follows

$$
\begin{aligned}
& \Lambda_{(5.2)}^{e *} z^{e *}(x)=\left\{\varepsilon \widetilde{a}^{e}(x) \delta_{\bar{x} \widehat{x}}+\widetilde{b}^{e}(x) \delta_{x}+\widetilde{c}^{e}(x)\right\} z^{e *}(x) \equiv \\
\equiv & \left(\widetilde{\Lambda}_{(5.4)}^{e p}+\widetilde{c}^{e}(x)\right) z^{e *}(x) \equiv \widetilde{\Lambda}_{(5.4)}^{e} z^{e *}(x), \quad x=x^{i} \in D_{h}^{e}, \quad i \notin I_{1} ; \\
& \Lambda_{(5.2)}^{e *} z^{e *}(x)=\widetilde{g}(x)\left(-z^{e *}(x)+z^{e *}\left(x^{j}\right)\right)+\widetilde{\beta}(x) z^{e *}(x) \equiv \\
\equiv & \left(\widetilde{\Lambda}_{(5.4)}^{e p}+\widetilde{\beta}(x)\right) z^{e *}(x) \equiv \widetilde{\Lambda}_{(5.4)}^{e} z^{e *}(x), \quad x=x^{i} \in D_{h}^{e}, \quad i \in I_{1}, \quad j=j_{(3.5)}(i),
\end{aligned}
$$


where $\widetilde{g}(x)=g_{i i}^{*}, \widetilde{\beta}(x)=g_{i j}^{*}-g_{i i}^{*}$.

We consider the case when the disturbed coefficients $q_{i j}^{e *}, g_{i j}^{*}$ are generated by the disturbed functions $a^{*}(x), b^{*}(x)$ and by the quantities $g^{*}\left(x^{i}\right)$, which correspond to the functions $a(x)$, $b(x), x \in D_{h}$ and $g\left(x^{i}\right), i \in I_{1}$, moreover, there are no other disturbances of $q_{i j}^{e}, g_{i j}$, except these ones.

For the disturbances of the values $g\left(x^{i}\right)$ we use the notation: $g^{\delta}\left(x^{i}\right)=g^{*}\left(x^{i}\right)-g\left(x^{i}\right)$.

2. We deduce some estimates for the disturbances of the data of scheme (5.2), (3.3), (3.16), which are generated by the disturbances of the data of the boundary value problem (2.1), (2.2).

2.1.For the coefficients of the operators $\widetilde{\Lambda}_{(5.4)}^{e}, \Lambda_{(5.2)}^{e *}$ we obtain the estimates

$$
\begin{aligned}
& \left|\widetilde{a}^{e}(x)-a^{e}(x)\right| \leq M_{1}\left|a^{e \delta}(x)\right|, \\
& \left|\widetilde{b}^{e}(x)-b^{e}(x)\right| \leq M_{2}\left[\widehat{\eta}_{1}(x)\left|a^{e \delta}(x)\right|+\left|b^{e \delta}(x)\right|\right], \\
& \left|\widetilde{c}^{e}(x)\right| \leq M_{3}\left[\widehat{\eta}_{2}(x)\left|a^{e \delta}(x)\right|+\widehat{\eta}_{3}(x)\left|b^{e \delta}(x)\right|\right], \quad x \in \widehat{D}_{h}^{e} ; \\
& \left|q_{i j}^{e \delta}\right| \leq M_{4}\left[\widehat{\eta}_{2}\left(x^{i}\right)\left|a^{e \delta}\left(x^{i}\right)\right|+\widehat{\eta}_{3}\left(x^{i}\right)\left|b^{e \delta}\left(x^{i}\right)\right|\right], \quad x^{i} \in \widehat{D}_{h}^{e} ; \\
& \widehat{\eta}_{1}(x)=\left\{N\left(\varepsilon+\ln ^{-1} N\right), \varepsilon N, \varepsilon N\right\}, \\
& \widehat{\eta}_{2}(x)=\left\{\varepsilon^{-1} N^{2}\left(\varepsilon+\ln ^{-1} N\right)^{2}, N^{2}\left(\varepsilon+\ln ^{-1} N\right), \varepsilon N^{2}\right\}, \\
& \widehat{\eta}_{3}(x)=\left\{\varepsilon^{-1} N\left(\varepsilon+\ln ^{-1} N\right), N, N\right\} ; \\
& |\widetilde{g}(x)-g(x)|,|\widetilde{\beta}(x)|,\left|g_{i j}^{\delta}\right| \leq M\left|g^{\delta}(x)\right|, \quad x=x^{i} \in \gamma_{h}^{e} ;
\end{aligned}
$$

here and below $\widehat{\eta}_{k}(x)=\widehat{\eta}_{k}(x ; \varepsilon, N)$ are piecewise constant functions in the vector notation $(5.1 \mathrm{c}) ; a^{e}(x)=a_{(3.4)}^{e}(x ; a(\cdot)), \ldots, b^{e \delta}(x)=b_{(3.4)}^{\delta e}\left(x ; b^{\delta}(\cdot)\right)$. Estimates (5.5) are unimprovable with respect to $a^{e \delta}(x), b^{e \delta}(x), g^{\delta}(x), \varepsilon, N$.

2.2. Let the disturbances satisfy the condition

$$
\begin{gathered}
\left|a^{e \delta}(x)\right| \leq m_{1} \widehat{\eta}(x), \quad\left|b^{e \delta}(x)\right| \leq m_{2}, \quad x \in \widehat{D}_{h}^{e} ; \quad\left|g^{\delta}(x)\right| \leq m_{3}, \quad x \in \gamma_{h}^{e} ; \\
\widehat{\eta}(x)=\left\{N^{-1}\left(\varepsilon+\ln ^{-1} N\right)^{-1},(1+\varepsilon N)^{-1},(1+\varepsilon N)^{-1}\right\} .
\end{gathered}
$$

In this case we have the estimate

$$
\begin{aligned}
& \left|\widetilde{a}^{e}(x)-a^{e}(x)\right|,\left|\widetilde{b}^{e}(x)-b^{e}(x)\right| \leq m, \quad x \in \widehat{D}_{h}^{e} ; \\
& |\widetilde{g}(x)-g(x)| \leq m, \quad x \in \gamma_{h}^{e},
\end{aligned}
$$

where $m=m_{(5.7)}\left(m_{1}, m_{2}, m_{3}\right) \rightarrow 0$ for $m_{i(5.6)} \rightarrow 0$. Under the condition (5.6) for the functions $\widetilde{c}^{e}(x), \widetilde{\beta}(x)$ we obtain the estimates

$$
\begin{gathered}
\left|\widetilde{c}^{e}(x)\right| \leq m \widehat{\eta}(x), \quad x \in \widehat{D}_{h}^{e}, \quad|\widetilde{\beta}(x)| \leq m, \quad x \in \gamma_{h}^{e}, \\
\widehat{\eta}(x)=\left\{\varepsilon^{-1} N\left(\varepsilon+\ln ^{-1} N\right), N+N^{2} \ln ^{-1} N(1+\varepsilon N)^{-1}, N\right\},
\end{gathered}
$$

where $m=m\left(m_{(5.6)}\right)$; the estimate $(5.8)$ is unimprovable with respect to $\varepsilon, N$.

2.3. In the case of sufficiently large values of $m_{1(5.6)}, m_{2(5.6)}, m_{3(5.6)}$ the coefficients $\widetilde{a}^{e}(x)$, $\widetilde{b}^{e}(x), \widetilde{g}(x)$ differ greatly from $a^{e}(x), b^{e}(x), g(x)$ and can be negative (in this case, generally speaking, the solution of problem (5.2), (3.16) does not exist). 
Definition. We say that the operator $\widetilde{\Lambda}_{(5.4)}^{e p}$, i.e., the main part of the operator $\widetilde{\Lambda}_{(5.4)}^{e}$, and the operator $\Lambda_{(3.5 c)}^{e}$ (its main part) are consistent with respect to the disturbances $a^{e \delta}(x), b^{e \delta}(x)$, $g^{\delta}(x)$ satisfying the condition (5.6) (or, shortly, with respect to the disturbances $a^{e \delta}(x), b^{e \delta}(x)$, $\left.g^{\delta}(x)\right)$ if their coefficients satisfy the estimate (5.7).

Lemma 5.1. Under the condition (5.6) the operators $\widetilde{\Lambda}_{(5.4)}^{e p}$ and $\Lambda_{(3.5)}^{e}$ are consistent with respect to the disturbances $a^{e \delta}(x), b^{e \delta}(x), g^{\delta}(x)$; for the coefficients $\widetilde{a}^{e}(x), \widetilde{b}^{e}(x), \widetilde{g}(x)$ (coefficients $\left.\widetilde{c}^{e}(x), \widetilde{\beta}(x)\right)$ under the condition (5.6) the estimate (5.7) (estimate (5.8)) is fulfilled.

3. We examine the existence of the solution of the perturbed difference scheme. Together with problem (5.2), (3.16) we consider a scheme which has the common with this problem operator $\widetilde{\Lambda}_{(5.4)}^{e p}$ as the main term in the discrete equations

$$
\begin{gathered}
\widetilde{\Lambda}_{(5.9)}^{e} \widetilde{z}^{e}(x) \equiv\left\{\varepsilon \widetilde{a}^{e}(x) \delta_{\bar{x} \widehat{x}}+\widetilde{b}^{e}(x) \delta_{x}+\widetilde{c}^{e}(x)\right\} \widetilde{z}^{e}(x)= \\
=\left(\widetilde{\Lambda}_{(5.4)}^{e p}+\widetilde{c}^{e}(x)\right) \widetilde{z}^{e}(x)=\widetilde{f}_{1}^{e}(x), \quad x \in \widehat{D}_{h}^{e}, \\
\widetilde{\Lambda}_{(5.9)}^{e} \widetilde{z}^{e}(x) \equiv \widetilde{g}(x)\left(-\widetilde{z}^{e}(x)+\widetilde{z}^{e}\left(x^{j}\right)\right)+\widetilde{\beta}(x) \widetilde{z}^{e}(x)= \\
=\left(\widetilde{\Lambda}_{(5.4)}^{e p}+\widetilde{\beta}(x)\right) \widetilde{z}^{e}(x)=\widetilde{f}_{1}^{e}(x), \quad x \in \gamma_{h}^{e}, \\
\widetilde{z}^{e}(x)=\widetilde{\varphi}^{e}(x), \quad x \in \Gamma_{h}^{e},
\end{gathered}
$$

where $\bar{D}_{h}^{e}=\bar{D}_{h}^{e}\left(\bar{D}_{h(3.16)}\right) ; \widetilde{c}_{(5.9)}^{e}(x), x \in \widehat{D}_{h}^{e}, \widetilde{\beta}(x)_{(5.9)}, x \in \gamma_{h}^{e}$ as opposed to $\widetilde{c}_{(5.4)}^{e}(x)$ and $\widetilde{\beta}_{(5.4)}(x)$ are independent of the data of problem (5.2), (3.16); in general, $\widetilde{c}_{(5.9)}^{e}(x) \neq \widetilde{c}_{(5.4)}^{e}(x), \widetilde{\beta}_{(5.9)}(x) \neq$ $\widetilde{\beta}_{(5.4)}(x)$ and $\widetilde{\Lambda}_{(5.9)}^{e} \neq \widetilde{\Lambda}_{(5.4)}^{e}=\Lambda_{(5.2)}^{e *}, x \in D_{h}^{e} ; \tilde{f}_{1}^{e}(x)=f_{1}^{e *}(x), x \in D_{h}^{e}, \widetilde{\varphi}^{e}(x)=\varphi^{e *}(x), x \in \Gamma_{h}^{e}$.

3.1. Note that under condition (5.6), in that case when the functions $\widetilde{c}_{(5.9)}^{e}(x), x \in \widehat{D}_{h}^{e}$ and $\widetilde{\beta}(x), x \in \gamma_{h}^{e}$ satisfy condition (5.8), the solution of problem (5.9), (3.16) does not exist in general.

The consideration of model examples shows that in the case of condition (5.6), if the following condition holds:

$$
\begin{gathered}
\widetilde{c}^{e}(x) \geq M \widehat{\eta}(x), \quad x \in \widehat{D}_{h}^{e}, \\
\widehat{\eta}(x)=\left\{\varepsilon^{-1}, N, N(1+\varepsilon N)^{-1}\right\} \\
|\widetilde{\beta}(x)| \geq M \eta^{\gamma}(x), \quad x \in \gamma_{h}^{e}, \\
\eta^{\gamma}(x)=\eta^{\gamma}(x ; \varepsilon, l)=\alpha(\varepsilon, l)\{1,1\}, \quad \alpha(\varepsilon, l)=l(\varepsilon+l)^{-1},
\end{gathered}
$$

where $M_{(5.10)}$ is sufficiently large, the solution of problem (5.9), (3.16) also, in general, does not exist. Note that $\alpha(\varepsilon, l) \geq h^{\gamma}\left(\varepsilon+h^{\gamma}\right)^{-1} \geq m N^{-1}$, where $h^{\gamma}$ is the stepsize of the mesh (3.16) on $\bar{D}^{(1)} \cap \bar{D}^{(2)}$. In the case of condition $(3.21 \mathrm{~b})$ we have $\alpha(\varepsilon, l) \geq m$.

3.2. The solution of problem (5.2), (3.16) exists for any $\varepsilon \in(0,1]$ and any $N$, if condition (5.6) and also the following condition are valid:

$$
\begin{aligned}
& \left|\widetilde{c}^{e}(x)\right| \leq m \widehat{\eta}_{(5.10)}(x), \quad x \in \widehat{D}_{h}^{e}, \\
& |\widetilde{\beta}(x)| \leq m \alpha_{(5.10)}(\varepsilon, l), \quad x \in \gamma_{h}^{e},
\end{aligned}
$$


where $m$ is sufficiently small. Such bounds for the functions $\widetilde{c}^{e}(x), \widetilde{\beta}(x)$ under the condition (5.6) (and condition (5.6) itself) hold if the values $a^{e \delta}(x), b^{e \delta(x)}, g^{\delta}(x)$ satisfy the condition

$$
\begin{aligned}
\left|a^{e \delta}(x)\right| & \leq m \widehat{\eta}_{1}(x), \quad\left|b^{e \delta}(x)\right| \leq m \widehat{\eta}_{2}(x), \quad x \in \widehat{D}_{h}^{e}, \quad m=m\left(m_{(5.11)}\right) \\
\widehat{\eta}_{1}(x) & =\left\{N^{-2}\left(\varepsilon+\ln ^{-1} N\right)^{-2}, N^{-1}\left(\varepsilon+\ln ^{-1} N\right)^{-1},(1+\varepsilon N)^{-2}\right\} \\
\widehat{\eta}_{2}(x) & =\left\{N^{-1}\left(\varepsilon+\ln ^{-1} N\right)^{-1}, 1,(1+\varepsilon N)^{-1}\right\} \\
\left|g^{\delta}(x)\right| & \leq m \alpha_{(5.10)}(\varepsilon, l), \quad x \in \gamma_{h}^{e} .
\end{aligned}
$$

Lemma 5.2. The conditions either (5.6), (5.11), or (5.12) are sufficient for the existence of the solutions of the difference scheme (5.2), (3.16). In the case of conditions (5.6), (5.10) the solution of the difference scheme (5.9), (3.16), in general, does not exist.

Remark 8. In order that the operators $\Lambda_{(3.5)}^{e}$ and $\widetilde{\Lambda}_{(5.4)}^{e p}$ are consistent and the solution of the difference scheme (5.2), (3.16) exists, the condition (5.12) is unimprovable with respect to $\varepsilon, N, l$; even for a sufficiently large value of $m_{(5.12)}$ the solution of difference scheme (5.2), (3.16) does not exist.

In Section 6 we determine conditions which ensure the $(\varepsilon, N)$-uniform boundedness of the solutions of scheme (5.2), (3.16). Note that the function $z^{e \delta}(x), x \in \bar{D}_{h}$, by virtue of (3.5) and (5.2), is the solution of the difference scheme

$$
\begin{aligned}
\widetilde{\Lambda}_{(5.4)}^{e} z^{e \delta}(x) & =f_{1}^{e \delta}(x)-\left(\widetilde{\Lambda}_{(5.4)}^{e}-\Lambda_{(3.5)}^{e}\right) z^{e}(x), \quad x \in D_{h}^{e}, \\
z^{e \delta}(x) & =\varphi^{e \delta}(x), \quad x \in \Gamma_{h}^{e},
\end{aligned}
$$

where $z^{e}(x)=z_{(3.5)}^{e}(x), x \in \bar{D}_{h}^{e}$ is the solution of problem (3.5), (3.16).

4. Before we proceed to the further study of problem (5.2), (3.16) (problem (5.13), (3.16)), we consider the simpler difference scheme

$$
\widetilde{\Lambda}_{(5.9)}^{e} \omega^{\delta}(x)=F^{\delta}(x), \quad x \in D_{h}^{e}, \quad \omega^{\delta}(x)=\varphi^{\delta}(x), \quad x \in \Gamma_{h}^{e} .
$$

4.1. Let the functions $\widetilde{c}_{(5.9)}(x), x \in \widehat{D}_{h}^{e}$ and $\widetilde{\beta}(x), x \in \gamma_{h}^{e}$ satisfy condition (5.11), and let the condition (5.6) hold for the coefficients $\widetilde{a}^{e}(x), \widetilde{b}^{e}(x), g(x)$. Then the solution of problem (5.14), (3.16) exists. Let also at least one of the following conditions be valid:

$$
\begin{gathered}
\left|F^{\delta}(x)\right|=\rho(\varepsilon, N) \eta(x), \quad x \in D_{h}^{e}, \quad \eta(x)=\left\{\widehat{\eta}(x), \eta^{\gamma}(x)\right\}, \\
\widehat{\eta}(x)=\left\{\varepsilon^{-1}\left(\varepsilon+\ln ^{-1} N\right), N, 1\right\}, \quad \eta^{\gamma}(x)=\eta_{(5.10)}^{\gamma}(x)=\alpha_{(5.10)}(\varepsilon, l)\{1,1\},
\end{gathered}
$$

where the quantity $\rho(\varepsilon, N)>0$ is not $(\varepsilon, N)$-uniformly bounded. Then the solution of the difference scheme (5.14), (3.16) (in particular, for $\left.\widetilde{c}^{e}(x) \equiv 0\right)$ is not $(\varepsilon, N)$-uniformly bounded.

4.2. Let the condition (5.6) hold for problem (5.14), (3.16), which ensures the proper structure of the operator $\left.\widetilde{\Lambda}_{(5.4)}^{e}\right)$, and also let the following condition be satisfied:

$$
\begin{aligned}
& \left|\widetilde{c}^{e}(x)\right| \leq m \widehat{\eta}_{(5.15)}(x), \quad x \in \widehat{D}_{h}^{e}, \quad|\widetilde{\beta}(x)| \leq m \eta_{(5.10)}^{\gamma}(x), \quad x \in \gamma_{h}^{e} ; \\
& \left|F^{\delta}(x)\right| \leq M \widehat{\eta}_{(5.15)}(x), \quad x \in \widehat{D}_{h}^{e}, \\
& \left|F^{\delta}(x)\right| \leq M \eta_{(5.10)}^{\gamma}(x), \quad x \in \gamma_{h}^{e}, \quad\left|\varphi^{\delta}(x)\right| \leq M, \quad x \in \Gamma_{h}^{e},
\end{aligned}
$$


where $m$ is sufficiently small, $m=m\left(m_{(5.11)}\right)$. Then the solution of the difference scheme (5.14), (3.16) is bounded $(\varepsilon, N)$-uniformly. Conditions (5.6), (5.16) are unimprovable with respect to $\varepsilon, l, N$ in order that the following properties are fulfilled: (a) the operators $\Lambda_{(3.5)}^{e}$ and $\widetilde{\Lambda}_{(5.4)}^{e p}$ are consistent with respect to the disturbances $a^{e \delta}(x), b^{e \delta}(x), g^{\delta}(x)$; (b) the solution of the difference scheme (5.14), (3.16) exists for any $\varepsilon \in(0,1]$ and $N$; (c) the solution of this scheme is bounded $(\varepsilon, N)$-uniformly. For example, for sufficiently large $m_{i(5.6)}, m_{(5.16)}$ the solution of the difference scheme, in general, does not exist or is not $(\varepsilon, N)$-uniformly bounded, while in the case $M_{(5.16)}=(o(1))^{-1}$ for $N \rightarrow \infty$ and/or $\varepsilon \rightarrow 0$ the solution of the difference scheme is not bounded $(\varepsilon, N)$-uniformly.

4.3. When conditions (5.6), (5.16a) are valid, the solution of the difference scheme (5.14), (3.16) satisfies the estimate

$$
\begin{aligned}
& \left|\omega^{\delta}(x)\right| \leq M\left[\max _{\widehat{D}_{h}^{e}}\left[\widehat{\eta}_{(5.15)}^{-1}(x)\left|F^{\delta}(x)\right|\right]+\right. \\
& \left.\quad+\max _{\gamma_{h}^{e}}\left[\left(\eta_{(5.10)}^{\gamma}(x)\right)^{-1}\left|F^{\delta}(x)\right|\right]+\max _{\Gamma_{h}^{e}}\left|\varphi^{\delta}(x)\right|\right], \quad x \in \bar{D}_{h}^{e} .
\end{aligned}
$$

This estimate is unimprovable with respect to $\varepsilon, l, N ; \widehat{\eta}_{(5.15)}(x)=\widehat{\eta}_{(5.15)}(x ; \varepsilon, N), \eta_{(5.10)}^{\gamma}(x)=$ $\eta_{(5.10)}^{\gamma}(x ; \varepsilon, l)$. Note that the quantities $\widehat{\eta}_{(5.15)}(x)$ and $\eta_{(5.10)}^{\gamma}(x)$ satisfy the lower bounds $\widehat{\eta}_{(5.15)}(x) \geq 1, x \in \widehat{D}_{h}^{e}, \eta_{(5.10)}^{\gamma}(x) \geq m N^{-1}, x \in \gamma_{h}^{e}$.

Theorem 5.1. Let the disturbances $a^{e \delta}(x), b^{e \delta}(x), x \in \widehat{D}_{h}^{e}, g^{\delta}(x), x \in \gamma_{h}^{e}$ of the difference scheme (3.5), (3.16) and the functions $\widetilde{c}_{(5.9)}^{e}(x), x \in \widehat{D}_{h}^{e}, \widetilde{\beta}_{(5.9)}(x), x \in \gamma_{h}^{e}$ satisfy the conditions (5.6), (5.16). Then the following statement is valid: the operators $\Lambda_{(3.5)}^{e}$ and $\widetilde{\Lambda}_{(5.4)}^{e p}$ are consistent with respect to the disturbances $a^{e \delta}(x), b^{e \delta}(x), x \in \widehat{D}_{h}^{e}, g^{\delta}(x), x \in \gamma_{h}^{e}$; the solution of the difference scheme (5.14), (3.16) exists for any $\varepsilon$ and $N$ and is $(\varepsilon, N)$-uniformly bounded; for this statement to be true the conditions (5.6), (5.16) are unimprovable with respect to $\varepsilon, l, N$. For the solutions of the difference scheme (5.14), (3.16), under the conditions (5.6), (5.16a) the unimprovable estimate (5.17) holds.

Remark 9. From estimate (5.17) it follows that the identical (in module) functions $F^{\delta}(x)$ from the different subdomains $D_{h}^{k}, k=1, \ldots, 5$ contribute differently to the function $\omega^{\delta}(x)$. One can observe the essential (with respect to the values of $\varepsilon, N$ ) anisotropy (over the subdomains $D_{h}^{k}$ ) concerning the influence of the right-hand side of the difference scheme (5.14), (3.16) on its solution. The function $\omega^{\delta}(x)$ can remain $\varepsilon$-uniformly bounded in the case of the functions $F^{\delta}(x)$ being not $\varepsilon$-uniformly bounded on $D_{h}^{1}$ (see condition (5.16b)). But, for the functions $F^{\delta}(x)$ bounded on $\gamma_{h}^{e}$, the function $\omega^{\delta}(x)$ generally increases without bound when $N$ grows, if condition (3.21b) fails.

\section{On conditioning of the difference scheme (5.2), (3.16)}

We consider the conditioning of the difference scheme (5.2), (3.16) obtained from scheme (3.5), (3.16) under disturbance of the data of the boundary value problem (2.1), (2.2).

1. As a preliminary, we investigate the conditioning of the difference scheme (5.14), (3.16).

1.1. To estimate the solution of scheme (5.14), (3.16), it is convenient to measure the function

$$
f(x)=f\left(x ; F^{\delta}(\cdot), \varphi^{\delta}(\cdot)\right) \equiv\left\{F^{\delta}(x), \varphi^{\delta}(x)\right\}, x \in \bar{D}_{h}^{e},
$$


where $f(x)=F^{\delta}(x), x \in D_{h}^{e}, f(x)=\varphi^{\delta}(x), x \in \Gamma_{h}^{e}$, i.e., the right-hand side of the discrete equations, in the special weight norm $\|\cdot\|^{S^{e}}$ :

$$
\begin{gathered}
\|f(x)\| \frac{S}{D}_{h}^{e}= \\
=\max \left\{\max _{\widehat{D}_{h}^{e}}\left[\widehat{\eta}_{(5.15)}^{-1}(x)\left|F^{\delta}(x)\right|\right], \max _{\gamma_{h}^{e}}\left[\left(\eta_{(5.10)}^{\gamma}(x)\right)^{-1}\left|F^{\delta}(x)\right|\right], \max _{\Gamma_{h}^{e}}\left|\varphi^{\delta}(x)\right|\right\} .
\end{gathered}
$$

Then the estimate (5.17) takes such a form

$$
\left|\omega^{\delta}(x)\right| \leq M\left\|f\left(x ; F^{\delta}(\cdot), \varphi^{\delta}(\cdot)\right)\right\|^{S^{e}}, \quad x \in \bar{D}_{h}^{e} .
$$

Thus, the difference scheme (5.14), (3.16) (scheme (5.2), (3.16)) can be regarded as a transformation which translates the discrete space with the maximum norm $\|\cdot\|$ to a discrete space endowed with the special norm $\|\cdot\|^{S^{e}}$, and so the quantity $\mathfrak{x}^{S^{e}}$ is considered as the condition number for this transformation.

1.2. The estimate (5.17) and the following estimate

$$
\begin{aligned}
& \max _{\left|\omega^{e}(\cdot)\right| \leq 1, D_{h}^{k}}\left|\widetilde{\Lambda}_{(5.9)}^{e} \omega^{e}(x)\right| \leq M \eta(x), x \in D_{h}^{k}, k=1, \ldots, 5 \\
& \eta(x)=\left\{\widehat{\eta}(x), \eta^{\gamma}(x)\right\}_{(6.1)}, \quad \eta^{\gamma}(x)=\{1,1\} \\
& \widehat{\eta}(x)=\left\{\varepsilon^{-1} N^{2}\left(\varepsilon^{2}+\ln ^{-2} N\right), N^{2}\left(\varepsilon+\ln ^{-1} N\right), N(1+\varepsilon N)\right\}
\end{aligned}
$$

imply the required estimates for the condition numbers $\mathfrak{x}^{S^{e}}\left(\widetilde{\Lambda}_{(5.9)}^{e}\right)$ and $\mathfrak{x}^{S^{e} k}\left(\widetilde{\Lambda}_{(5.9)}^{e}\right)$, where

$$
æ^{S^{e} k}\left(\widetilde{\Lambda}_{(5.9)}^{e}\right)=\max _{\left|\omega^{e}(\cdot)\right| \leq 1, D_{h}^{k}}\left[\left|\widetilde{\Lambda}_{(5.9)}^{e} \omega^{e}(x)\right| \eta_{(6.1)}^{-1}(x)\right], \quad k=1, \ldots, 5
$$

are the fractional condition numbers of the operator $\widetilde{\Lambda}_{(5.9)}^{e}$ of problem (5.14), (3.16). We thus have the following estimates:

$$
\begin{aligned}
& \mathfrak{x}^{S^{e}}\left(\widetilde{\Lambda}_{(5.9)}^{e}\right) \leq M \eta^{1} ; \mathfrak{x}^{S^{e} k}\left(\widetilde{\Lambda}_{(5.9)}^{e}\right) \leq M \eta^{2}(x), \quad x \in D_{h}^{k}, k=1, \ldots, 5, \\
& \eta^{1}=\eta^{1}(\varepsilon, N)=\max \left[N^{2}\left(\varepsilon+\ln ^{-1} N\right), \alpha_{(5.10)}^{-1}(\varepsilon, l)\right]=N^{2}\left(\varepsilon+\ln ^{-1} N\right) \leq N^{2} \\
& \eta^{2}(x)=\left\{\widehat{\eta}^{2}(x), \eta^{\gamma}(x)\right\}, \quad \eta^{\gamma}(x)=\alpha_{(5.10)}^{-1}(\varepsilon, l)\{1,1\} \\
& \widehat{\eta}^{2}(x)=\left\{N^{2}\left(\varepsilon+\ln ^{-1} N\right), N\left(\varepsilon+\ln ^{-1} N\right), N(1+\varepsilon N)\right\}
\end{aligned}
$$

The estimates for $\mathfrak{x}^{S^{e}}, \mathfrak{x}^{S^{e} k}$ are unimprovable with respect to $\varepsilon, N, l$.

In the case of scheme (5.14), (3.16) a small relative disturbance of the right-hand side and the boundary conditions in the special norm (i.e., the small quantity $\left\|f_{0}^{\delta}(x)\right\|^{S^{e}}\left(\left\|f_{0}(x)\right\|^{S^{e}}\right)^{-1}$, where $\left.f_{0}(x)=\left\{F_{0}(x), \varphi_{0}(x)\right\}, F_{0}(x)=F_{(5.14)}^{\delta}(x), \varphi_{0}(x)=\varphi_{(5.14)}^{\delta}(x)\right)$ in the case of condition (5.16a) causes (stipulates) a small relative disturbance of the solution of the difference scheme in the maximum discrete norm (i.e., the smallness of the quantity $\left\|\omega_{0}^{\delta}(x)\right\|\left(\left\|\omega_{0}(x)\right\|\right)^{-1}$, $\left.\omega_{0}(x)=\omega_{(5.14)}^{\delta}(x)\right)$. Thus, the difference scheme (5.14), (3.16), in the case of the special norm, is well conditioned $\varepsilon$-uniformly; the conditioning of the difference scheme differs essentially over the subsets $D_{h}^{k}, k=1, \ldots, 5$. 
Lemma 6.1. Let the disturbances $a^{e \delta}(x), b^{e \delta}(x), x \in \widehat{D}_{h}^{e}, g^{\delta}(x), x \in \gamma_{h}^{e}$ of the difference scheme (3.5), (3.16) and the functions $\widetilde{c}_{(5.9)}^{e}(x), x \in \widehat{D}_{h}^{e}, \widetilde{\beta}_{(5.9)}(x), x \in \gamma_{h}^{e}$ satisfy the conditions (5.6), (5.16a). Then the difference scheme (5.14), (3.16) is well conditioned $\varepsilon$-uniformly in the case of the special norm $\|\cdot\|_{(6.1)}^{S^{e}} ;$ for the condition numbers $\mathfrak{x}^{S^{e}}\left(\widetilde{\Lambda}_{(5.9)}^{e}\right)$ and $\mathfrak{x}^{S^{e} k}\left(\widetilde{\Lambda}_{(5.9)}^{e}\right)$ the estimates (6.2) hold.

Remark 10. Having in mind estimate (5.17), i.e., the dependence of the solution of the difference scheme (5.14), (3.16) on the right-hand side $F^{\delta}(x), x \in D_{h}$, it seems appropriate to write the difference scheme (5.14), (3.16) such that the solution of the difference scheme depends on the right-hand side "identically" on the subsets $D_{h}^{k}, k=1, \ldots, 5$. Multiplying the difference equations in (5.14), which correspond to $x^{i} \in D_{h}^{1}, x^{i} \in D_{h}^{2}$ and $x^{i} \in \gamma_{h}^{e}$, by the values $\varepsilon\left(\varepsilon+\ln ^{-1} N\right)^{-1}, N^{-1}$ and $\alpha_{(5.10)}^{-1}(\varepsilon, l)$ respectively, we obtain

$$
\begin{aligned}
& \Lambda^{S^{1}} \omega^{\delta}(x) \equiv\left\{\varepsilon a^{S^{1}}(x) \delta_{\widehat{x} \widehat{x}}+b^{S^{1}}(x) \delta_{x}+c^{S^{1}}(x)\right\} \omega^{\delta}(x)=F^{S^{1}}(x), \quad x \in \widehat{D}_{h}^{e}, \\
& \Lambda^{S^{1} \omega^{\delta}(x)} \equiv g^{S^{1}}(x)\left(-\omega^{\delta}(x)+\omega^{\delta}\left(x^{j}\right)\right)+\beta^{S^{1}}(x) \omega^{\delta}(x)=F^{S^{1}}(x), \quad x=x^{i} \in \gamma_{h}^{e}, j=j_{(3.5)}(i), \\
& \omega^{\delta}(x)=\varphi^{\delta}(x), \quad x \in \Gamma_{h}^{e} .
\end{aligned}
$$

Here $v^{S^{1}}(x)=g(x) \widetilde{v}(x), \widetilde{v}(x)$ is one of the functions $\widetilde{a}^{e}(x), \widetilde{b}^{e}(x), \widetilde{c}^{e}(x), F^{\delta}(x), x \in D_{h}^{e}$, or $\widetilde{g}(x)$, $\widetilde{\beta}(x), x \in \gamma_{h}^{e} ; g(x)=\eta_{(5.15)}^{-1}(x ; \varepsilon, N)$. The solution of the difference scheme (6.3), (3.16) under conditions (5.6), (5.16a) satisfies the estimate

$$
\left|\omega^{\delta}(x)\right| \leq M \max \left\{\max _{D_{h}^{e}}\left|F^{S^{1}}(x)\right|, \max _{\Gamma_{h}^{e}}\left|\varphi^{\delta}(x)\right|\right\}, x \in \bar{D}_{h}^{e},
$$

for the condition number of the discrete operator $\Lambda_{(6.3)}^{S^{1}}$ (obtained by "preconditioning" of the operator $\Lambda_{(5.9)}^{e}$ ) we have the estimate

$$
æ\left(\Lambda_{(6.3)}^{S^{1}}\right) \leq M \eta_{(6.2)}^{1}(\varepsilon, N)
$$

2. Let us estimate the solution of the difference scheme (5.13), (3.16).

Conditions (5.16a) and (5.6), which ensure the consistency of the operators $\Lambda_{(3.5)}^{e}$ and $\widetilde{\Lambda}_{(5.4)}^{e p}$ with respect to the disturbances $a^{e \delta}(x), b^{e \delta}(x), x \in \widehat{D}_{h}^{e}, g^{\delta}(x), x \in \gamma_{h}^{e}$ and the existence of the solution of the difference scheme (5.13), (3.16) (scheme (5.2), (3.16)), are valid if the disturbances $a^{e \delta}(x), b^{e \delta}(x), g^{\delta}(x)$ satisfy the relations

$$
\begin{aligned}
\left|a^{e \delta}(x)\right| & \leq m \widehat{\eta}_{1}(x), \quad\left|b^{e \delta}(x)\right| \leq m \widehat{\eta}_{2}(x), \quad x \in \widehat{D}_{h}^{e}, \\
\widehat{\eta}_{1}(x) & =\left\{N^{-2}\left(\varepsilon+\ln ^{-1} N\right)^{-1}, N^{-1}\left(\varepsilon+\ln ^{-1} N\right)^{-1},\left(1+\varepsilon N^{2}\right)^{-1}\right\}, \\
\widehat{\eta}_{2}(x) & =\left\{N^{-1}, 1, N^{-1}\right\}, \\
\left|g^{\delta}(x)\right| & \leq m \eta_{(5.10)}^{\gamma}(x), \quad x \in \gamma_{h}^{e},
\end{aligned}
$$

where $m$ is sufficiently small. But if the value $m$ is sufficiently large at least on one of the sets $D_{h}^{k}, k=1, \ldots, 5$, then at least one of the conditions (5.6) or (5.16a) fails; the condition (6.4) is unimprovable with respect to $\varepsilon, N$. 
Using the maximum principle, under conditions (5.6), (5.16a) (condition (6.4)) we establish the following estimate for the solutions of the difference scheme (5.13), (3.16):

$$
\begin{aligned}
& \left|z^{e \delta}(x)\right| \leq M\left\{\left\{\max _{\widehat{D}_{h}^{e}}\left\{\widehat{\eta}_{(5.15)}^{-1}(x)\left[\widehat{\eta}_{2(5.5)}(x)\left|a^{e \delta}(x)\right|+\widehat{\eta}_{3(5.5)}(x)\left|b^{e \delta}(x)\right|\right]\right\}+\right.\right. \\
& \left.\quad+\max _{\gamma_{h}^{e}}\left[\left(\eta_{(5.10)}^{\gamma}(x)\right)^{-1} g^{\delta}(x)\right]\right\} \max _{\bar{D}_{h}^{e}}\left|z^{e}(x)\right|+\max _{\widehat{D}_{h}^{e}}\left[\widehat{\eta}_{(5.15)}^{-1}(x)\left|f^{e \delta}(x)\right|\right]+ \\
& \left.\quad+\max _{\gamma_{h}^{e}}\left[\left(\eta_{(5.10)}^{\gamma}(x)\right)^{-1}\left|f^{e \delta}(x)\right|\right]+\max _{\Gamma_{h}^{e}}\left|\varphi^{e \delta}(x)\right|\right\}, \quad x \in \bar{D}_{h}^{e} .
\end{aligned}
$$

The condition numbers of the operator $\Lambda_{(5.2)}^{e *}$ of the difference scheme (5.13), (3.16) (scheme (5.2), (3.16)) in the case of the special norm satisfy the estimates

$$
\begin{aligned}
& \Re^{S^{e}}\left(\Lambda_{(5.2)}^{e *}\right)=\Re^{S^{e}}\left(\widetilde{\Lambda}_{(5.4)}^{e}\right) \leq M \eta_{(6.2)}^{1}(\varepsilon, N), \\
& \Re^{S^{e} k}\left(\Lambda_{(5.2)}^{e *}\right)=\Re^{S^{e} k}\left(\widetilde{\Lambda}_{(5.4)}^{e}\right) \leq M \eta_{(6.2)}^{2}(x ; \varepsilon, N, l), \quad x \in D_{h}^{k}, \quad k=1, \ldots, 5 .
\end{aligned}
$$

Estimates (6.5), (6.6) are unimprovable with respect to $\varepsilon, N, l$. Note that the $\varepsilon$-uniform estimate for $æ^{S^{e}}\left(\Lambda_{(5.2)}^{e *} ; \bar{D}_{h(3.16)}\right)$ under conditions $(5.6)$, (5.16a) is similar to estimate (3.9), which is the estimate for $æ(A)=æ\left(\Lambda_{(2.4)} ; \bar{D}_{h(3.7)}\right)$.

In the case of condition (6.4) and also the condition

$$
\left|f^{e \delta}(x)\right| \leq M \eta_{(5.15)}(x), \quad x \in D_{h}^{e}, \quad\left|\varphi^{e \delta}(x)\right| \leq M, \quad x \in \Gamma_{h}^{e}
$$

the solution of the difference scheme $(5.13),(3.16)$ is $(\varepsilon, N)$-uniformly bounded.

3. The consistency of the operators $\Lambda_{(3.5)}^{e}$ and $\widetilde{\Lambda}_{(5.4)}^{e p}$ with respect to the disturbances $a^{e \delta}(x)$, $b^{e \delta}(x), g^{\delta}(x)$ does not imply the monotonicity of the perturbed difference scheme (5.13), (3.16). For the consistent operators $\Lambda_{(3.5)}^{e}$ and $\widetilde{\Lambda}_{(5.4)}^{e p}$ the condition

$$
\begin{aligned}
& q_{i i}^{e}\left(a_{i}^{e *}\left(x^{i}\right), b_{i}^{e *}\left(x^{i}\right)\right) \geq q_{i i}^{e}\left(a^{e}\left(x^{i}\right), b^{e}\left(x^{i}\right)\right), \\
& q_{i j}^{e}\left(a_{j}^{e *}\left(x^{i}\right), b_{j}^{e *}\left(x^{i}\right)\right) \leq q_{i j}^{e}\left(a^{e}\left(x^{i}\right), b^{e}\left(x^{i}\right)\right), \quad x^{i} \in \widehat{D}_{h}^{e}, \quad j \neq i, \\
& g_{i i}^{*} \geq g\left(x^{i}\right), \quad g_{i j}^{*} \leq g\left(x^{j}\right), \quad x^{i} \in \gamma_{h}^{e}, \quad j \neq i
\end{aligned}
$$

is sufficient for the $(\varepsilon, N)$-uniform monotonicity of scheme (5.2), (3.16) (scheme (5.13), (3.16)). If at least one of the inequalities (for some values of $\varepsilon$ and $N$ ) fails, this leads generally to a loss of $(\varepsilon, N)$-uniform monotonicity of the difference scheme.

The condition

$$
\begin{aligned}
& q_{i i}^{e *}\left(a_{i}^{e *}\left(x^{i}\right), b_{i}^{e *}\left(x^{i}\right)\right)>0, \quad q_{i j}^{e *}\left(a_{j}^{e *}\left(x^{i}\right), b_{j}^{e *}\left(x^{i}\right)\right) \geq 0, \\
& q_{i i}^{e *}\left(a_{i}^{e *}\left(x^{i}\right), b_{i}^{e *}\left(x^{i}\right)\right)-q_{i, i-1}^{e *}\left(a_{i-1}^{e *}\left(x^{i}\right), b_{i-1}^{e *}\left(x^{i}\right)\right)- \\
& \quad-q_{i, i+1}^{e *}\left(a_{i+1}^{e *}\left(x^{i}\right), b_{i+1}^{e *}\left(x^{i}\right)\right) \geq 0, \quad x^{i} \in \widehat{D}_{h}^{e}, \quad j \neq i ; \\
& g_{i i}^{*} \geq g_{i j}^{*}>0, \quad x^{i} \in \gamma_{h}^{e}, \quad j \neq i
\end{aligned}
$$

is sufficient for the $(\varepsilon, N)$-uniform monotonicity of scheme (5.13), (3.16) even if the operators $\Lambda_{(3.5)}^{e}, \widetilde{\Lambda}_{(5.4)}^{e p}$ are not assumed to be consistent. The condition (6.9) is unimprovable. 
Theorem 6.1. Let the disturbances of the data of the boundary value problem (2.1), (2.2) satisfy the conditions (5.6), (5.16a) (condition (6.4)). Then the following statements are valid: (a) the operators $\Lambda_{(3.5)}^{e}$ and $\widetilde{\Lambda}_{(5.4)}^{e p}$ are consistent with respect to the disturbances $a^{e \delta}(x), b^{e \delta}(x)$, $x \in \widehat{D}_{h}^{e}, g^{\delta}(x), x \in \gamma_{h}^{e}$, moreover, the solution of the difference scheme (5.2), (3.16) exists for any $\varepsilon \in(0,1]$ and $N$; for the statement (a) to be true the conditions (5.6), (5.16a) (condition (6.4)) are unimprovable with respect to $\varepsilon, N$; (b) the condition numbers of the difference scheme (5.2), (3.16) and the solutions of the difference scheme (5.13), (3.16) satisfy the estimates (6.5), (6.6) which are unimprovable with respect to $\varepsilon, N$, l; the condition (6.7) is necessary and sufficient for the solution of the difference scheme (5.2), (3.16) to be $(\varepsilon, N)$-uniformly bounded; (c) under the condition (6.8) (except the conditions (5.6), (5.16a) or the condition (6.4)) the scheme (5.2), (3.16) is ( $\varepsilon, N)$-uniformly monotone.

Remark 11. The scheme (5.2), (3.16) is $(\varepsilon, N)$-uniformly monotone if condition (6.9) is satisfied (the fulfillment of conditions (5.6), (5.16a) or condition (6.4) is not assumed).

Remark 12. Let the hypothesis of Theorems 3.3, 6.1 be fulfilled. Then we have the estimate

$$
\begin{aligned}
& \left|u^{e}(x)-z^{e *}(x)\right| \leq M\left\{N^{-1} \ln N+\left\{\operatorname { m a x } _ { \widehat { D } _ { h } ^ { e } } \left\{\widehat { \eta } _ { ( 5 . 1 5 ) } ^ { - 1 } ( x ) \left[\widehat{\eta}_{2(5.5)}(x)\left|a^{e \delta}(x)\right|+\right.\right.\right.\right. \\
& \left.\left.\left.+\widehat{\eta}_{3(5.5)}(x)\left|b^{e \delta}(x)\right|\right]\right\}+\max _{\gamma_{h}^{e}}\left[\left(\eta_{(5.10)}^{\gamma}(x)\right)^{-1} g^{\delta}(x)\right]\right\} \max _{\bar{D}_{h}^{e}}\left|z^{e}(x)\right|+ \\
& \left.\quad+\max _{\widehat{D}_{h}^{e}}\left[\widehat{\eta}_{(5.15)}^{1}(x)\left|f^{e \delta}(x)\right|\right]+\max _{\gamma_{h}^{e}}\left[\left(\eta_{(5.10)}^{\gamma}(x)\right)^{-1}\left|f^{e \delta}(x)\right|\right]+\max _{\Gamma_{h}^{e}}\left|\varphi^{e \delta}(x)\right|\right\}, \quad x \in \bar{D}_{h}^{e} .
\end{aligned}
$$

Remark 13. Let the following condition be valid:

$$
|u(x)| \leq M, \quad x \in \bar{D}_{h} .
$$

If we disturb the data of the boundary value problem $(2.1),(2.2)$, the conditions

$$
\begin{aligned}
& \left|a^{e \delta}(x)\right|=o\left(\widehat{\eta}_{1(6.4)}(x)\right), \quad\left|b^{e \delta}(x)\right|=o\left(\widehat{\eta}_{2(6.4)}(x)\right), \quad x \in \widehat{D}_{h}^{e} ; \\
& \left|g^{\delta}(x)\right|=o\left(\eta_{(5.10)}^{\gamma}(x)\right), \quad x \in \gamma_{h}^{e}, \\
& \left|f^{e \delta}(x)\right|=o\left(\eta_{(5.15)}(x)\right), \quad x \in D_{h}^{e}, \\
& \left|\varphi^{e \delta}(x)\right|=o(1), \quad x \in \Gamma_{h}^{e} \text { for } \quad N \rightarrow \infty, \quad \varepsilon \in(0,1],
\end{aligned}
$$

where the value $o(\cdot)$ is uniformly in $\varepsilon$ small for $N \rightarrow \infty$, are sufficient for the $\varepsilon$-uniform convergence of the solutions of the difference scheme (5.2), (3.16) to the solution of the boundary value problem $(2.1),(2.2)$. The sufficient conditions in the case of the disturbances $a^{e \delta}(x)$, $x \in \widehat{D}_{h}^{e}$ and $g^{\delta}(x), x \in \gamma_{h}^{e}$ become less restrictive for small values of the parameter $\varepsilon$; for example, for $\varepsilon \leq M N^{-1}$ in the case of condition (3.21b), these conditions for $a^{e \delta}(x)$ and $g^{\delta}(x)$ take the form

$$
\begin{aligned}
\left|a^{e \delta}(x)\right| & =o(\widehat{\eta}(x)), \quad x \in \widehat{D}_{h}^{e}, \quad\left|g^{\delta}(x)\right|=o(1), \quad x \in \gamma_{h}^{e}, \\
N \rightarrow \infty, \quad \varepsilon \in\left(0, M N^{-1}\right], \quad \widehat{\eta}(x) & =\left\{N^{-2} \ln N, N^{-1} \ln N, N^{-1}\right\} .
\end{aligned}
$$

The estimates (6.10)-(6.13) allows us to choose appropriate accuracy for calculating $a(x)$, $b(x), f(x), x \in D_{h}$ on the subdomains $D_{h}^{k}, k=1, \ldots, 5$ and the number of mesh points which 
ensures the required accuracy of the numerical solution (computed on an absolutely exact computer).

Remark 14. The technique given in the paper allows us to investigate the conditioning of the Schwarz method in the case of a boundary value problem for the equation

$$
L u(x) \equiv\left\{\varepsilon a(x) \frac{d^{2}}{d x^{2}}+b(x) \frac{d}{d x}-c(x)\right\} u(x)=f(x), \quad x \in D .
$$

\section{References}

[1] N.S. Bakhvalov, On the optimization of methods for boundary-value problems with boundary layers, Zh. Vychisl. Mat. Mat. Fiz., 9 (1969), No. 4, pp. 841-859 (in Russian).

[2] A.M. Il'in, Differencing scheme for a differential equation with a small parameter affecting the highest derivative, Math. Notes, 6, (1969), No. 2, pp. 596-602.

[3] E.P. Doolan, J.J.H. Miller and W.H.A. Schilders, Uniform Numerical Methods for Problems with Initial and Boundary Layers, Boole Press, Dublin, 1980.

[4] G. I. Shishkin, Grid Approximations of Singularly Perturbed Elliptic and Parabolic Equations, Ural Branch of Russian Acad. Sci., Ekaterinburg, 1992 (in Russian).

[5] H.-G. Roos, M. Stynes and L. Tobiska, Numerical Methods for Singularly Perturbed Differential Equations: Convection-Diffusion and Flow Problems, Springer-Verlag, Berlin, 1996.

[6] J. J. H. Miller, E. O'Riordan, and G. I. Shishkin, Fitted Numerical Methods for Singular Perturbation Problems. Error Estimates in the Maximum Norm for Linear Problems in One and Two Dimensions, World Scientific, Singapore, 1996.

[7] A. A. Samarskii, Theory of Difference Schemes, 3rd edn., Nauka, Moscow, 1989 (in Russian); English transl.: The Theory of Difference Schemes, Marcel Dekker, Inc., New York, 2001.

[8] H.-G. Roos, A note on the conditioning of upwind shemes on Shishkin meshes, IMA J. Numer. Anal., 16 (1996), No. 4, pp. 529-538.

[9] A.M. Il'in, A.S. Kalashnikov, O.A. Oleinik, Linear equations of the second order of parabolic type, Uspehi Mat. Nauk, 17 (1962), pp. 3-146 (in Russian).

[10] N.S. Bakhvalov, Numerical methods, Moscow, Nauka, 1973 (in Russian).

[11] Axelsson O., Iterative Solution Methods, Cambridge University Press, 1994.

[12] G. I. Shishkin, Grid approximation of singularly perturbed equations with convective terms under perturbation of the data, Comput. Maths. Math. Phys., 41 (2001), No. 5, pp. 649-664. 